(C) 2019 IEEE. Personal use of this material is permitted. Permission from IEEE must be obtained for all other uses, in any current or future media, including reprinting/republishing this material for advertising or promotional purposes, creating new collective works, for resale or redistribution to servers or lists, or reuse of any copyrighted component of this work in other works.

This is the authors' manuscript which is accepted for publication in the IEEE Transactions on Robotics.

Digital Object Identifier (DOI): 10.1109/TRO.2019.2945876 


\title{
Hierarchical Impedance-based Tracking Control of Kinematically Redundant Robots
}

\author{
Alexander Dietrich and Christian Ott
}

\begin{abstract}
The control of a robot in its task space is a standard approach nowadays. If the system is kinematically redundant with respect to this goal, one can even execute additional subtasks simultaneously. By utilizing null space projections, for example, the whole stack of tasks can be implemented within a strict task hierarchy following the order of priority. One of the most common methods to track multiple task-space trajectories at the same time is to feedback-linearize the system and dynamically decouple all involved subtasks, which finally yields exponential stability of the desired equilibrium. Here, we provide a hierarchical multi-objective controller for trajectory tracking that ensures both asymptotic stability of the equilibrium and a desired contact impedance at the same time. In contrast to the state of the art in prioritized multi-objective control, feedback of the external forces can be avoided and the natural inertia of the robot is preserved. The controller is evaluated in simulations and on a standard lightweight robot with torque interface. The approach is predestined for precise trajectory tracking where dedicated and robust physical-interaction compliance is crucial at the same time.
\end{abstract}

Index Terms-Force control, redundant robots, hierarchical control, impedance control, physical human-robot interaction, whole-body control

\section{INTRODUCTION}

The definition and execution of tasks in the operational (or task) space is one of the most common approaches in robotics. While this strategy both applies to kinematic and force-torque control, especially the latter one is getting more and more important in the emerging field of physical human-robot interaction these days. If the robot is kinematically redundant with respect to the given task, it is possible to perform additional subtasks simultaneously as exemplified in Fig. 1. A common approach is to execute them in a prioritized manner by means of null space projections which have already been introduced to robotics decades ago [1]-[3] and have become standard tools since. One can ensure that lower-priority subtasks do not disturb the execution of more important ones. To stay with the example in Fig. 1, one could assign a task hierarchy with safety-critical objectives such as collision avoidance having high priority. As a result, subtasks with lower priorities, e. g., the optimization of the joint configuration, would consequently not compromise safety.

In recent years multi-objective control has also been tackled successfully by solving numerical optimization problems [4] [7]. The main advantage of these methods is that inequality constraints can be straightforwardly integrated into the task hierarchy. However, analyzing the stability properties is rather

The authors are with the Institute of Robotics and Mechatronics, German Aerospace Center (DLR), Wessling, Germany, alexander.dietrich@dlr.de

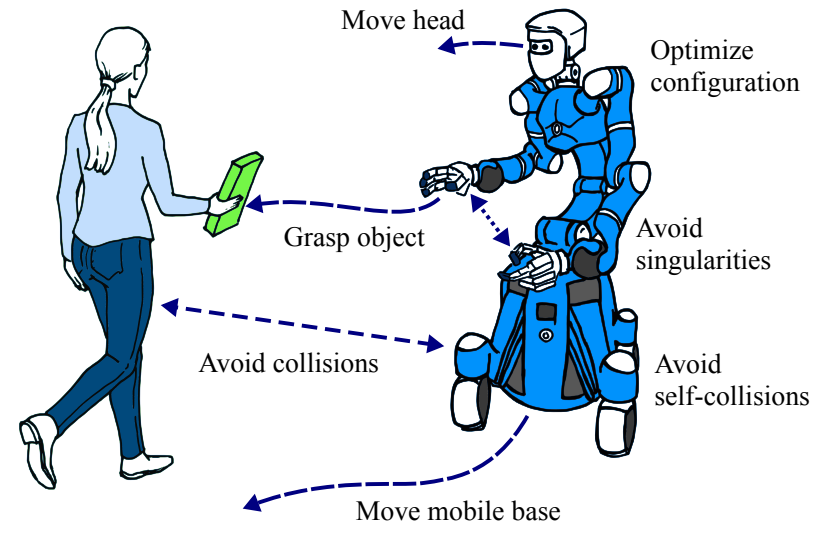

Fig. 1. A motivational example of multi-tasking in kinematically redundant robots. The system performs several subtasks simultaneously.

difficult, especially in the presence of these inequality constraints. But for the use of robots in the vicinity of humans, it is crucial to ensure stability in any case.

In contrast to multi-objective control approaches utilizing strict task hierarchies, one can alternatively apply soft priorities instead, for example by weighting the subtasks and superposing their control actions [8]-[11]. Continuously changing the weights endows the robot with the ability to switch the order or priority online in a smooth way. However, soft priorities inevitably introduce a coupling between the tasks such that these compete with each other in one way or another.

Among the approaches for strict hierarchies the so-called Operational Space Formulation (OSF) is probably the most prominent one [12] which is applied frequently in the literature. A feedback linearization is performed on all hierarchy levels and linear equations of motion are enforced by the controller. In order to prevent lower-level tasks from disturbing more important ones, dynamic consistency [13] is utilized, which can be interpreted as an energetical decoupling of the hierarchy levels. In [14] it has been shown that OSF approaches such as [15], [16] and common inverse dynamics solutions are in fact equivalent due to feedback linearization. If all subtasks are simultaneously feasible and independent, one can straightforwardly show exponential stability on the basis of the linear closed-loop dynamics in the OSF. However, that requires accurate knowledge of all dynamic parameters and the problematic feedback of all external forces and torques in the control if a desired task-space compliance is supposed to be realized.

In general, the analysis of stability for hierarchical controllers is known to be difficult [17] and an active field of research. Nevertheless, several aspects have already been addressed 
successfully. The quadratic-programming framework [11] has been proven stable both numerically and dynamically without inequality constraints. However, since the approach is weightprioritized, the task hierarchy is not strict by definition. For kinematic multi-priority control a formal stability analysis has been presented in [18]. Recently, the acceleration-based framework [19] was investigated in terms of stability. The proof of asymptotic stability covers tracking tasks on all but the lowest hierarchy level where compliance is realized. However, the approach does not allow to specify the contact behavior explicitly by setting the desired stiffness in the task-space. Moreover, the proof imposes restrictions on the feedback gains. In general, Lyapunov functions are frequently used in the control design and stability analysis of task hierarchies [5], [12], [18]-[24]. The term compliance control describes a variation of classical impedance control [25] in which the natural inertia is preserved and the desired contact stiffness can be explicitly specified. For a set of hierarchically arranged compliance control tasks asymptotic stability of the equilibrium has already been shown [23], [24]. The main advantage is that external forces and torques are not required in the feedback which is highly beneficial in terms of robustness. The drawback is the restriction of the controller to regulation tasks. In [21], a controller-observer approach for physical interaction with the environment was presented. In the two-level hierarchy the task-space error convergence is ensured while compliant null space behavior is achieved simultaneously. Theory from conditional stability is utilized for the analysis of the dynamics but an observation of the external forces and torques is necessary.

In this work the hierarchical compliance control approach for strict task hierarchies with an arbitrary number of priority levels is completed by incorporating trajectory tracking on all levels and providing the mathematical proof of uniform asymptotic stability of the equilibrium. A special feature of the presented approach is that the control law simplifies to the already established hierarchical regulation controller [24] if the desired task-space trajectories are stopped. Beside trajectory tracking, the presented controller realizes the desired contact stiffness and damping. In contrast to previous works where LaSalle's invariance principle could be exploited due to time invariance of the problem, stability theorems for nonautonomous systems have to be applied for the considered case of trajectory tracking [26], [27]. The theoretical findings are validated in simulations and compared with the state of the art. Moreover, the presented controller is evaluated and confirmed in experiments on a standard torque-controlled lightweight robot, namely a KUKA LWR IV+.

The article is organized as follows: After the recapitulation of projection-based, hierarchical task decoupling in Section II, the control law is derived in Section III. In Section IV the stability analysis is conducted, followed by the discussion in Section V. Afterwards, simulations in Section VI and experiments in Section VII validate the approach and compare it with the state of the art before the article is concluded in Section VIII.

\section{FUNDAMENTALS AND PRELIMINARIES}

The equations of motion of a robot with $n$ degrees of freedom (DOF) can be described by

$$
\boldsymbol{M}(\boldsymbol{q}) \ddot{\boldsymbol{q}}+\boldsymbol{C}(\boldsymbol{q}, \dot{\boldsymbol{q}}) \dot{\boldsymbol{q}}+\boldsymbol{g}(\boldsymbol{q})=\boldsymbol{\tau}+\boldsymbol{\tau}^{\mathrm{ext}},
$$

where $\boldsymbol{q}, \dot{\boldsymbol{q}}, \ddot{\boldsymbol{q}} \in \mathbb{R}^{n}$ denote the joint configuration, velocity, and acceleration, respectively. The inertia matrix $\boldsymbol{M}(\boldsymbol{q}) \in \mathbb{R}^{n \times n}$ is symmetric and positive definite, the Coriolis/centrifugal matrix $\boldsymbol{C}(\boldsymbol{q}, \dot{\boldsymbol{q}}) \in \mathbb{R}^{n \times n}$ is formulated such that $\dot{\boldsymbol{M}}(\boldsymbol{q}, \dot{\boldsymbol{q}})=\boldsymbol{C}(\boldsymbol{q}, \dot{\boldsymbol{q}})+\boldsymbol{C}(\boldsymbol{q}, \dot{\boldsymbol{q}})^{T}$ holds [28], and the generalized gravity forces are described by $\boldsymbol{g}(\boldsymbol{q}) \in \mathbb{R}^{n}$. The generalized forces $\tau \in \mathbb{R}^{n}$ in (1) determine the control input, while $\tau^{\text {ext }} \in \mathbb{R}^{n}$ represents the generalized external forces. The standard assumption is made that $\boldsymbol{M}(\boldsymbol{q})$ and $\boldsymbol{C}(\boldsymbol{q}, \dot{\boldsymbol{q}})$ are bounded for all possible values of $\boldsymbol{q}$ [22], and $\boldsymbol{C}(\boldsymbol{q}, \dot{\boldsymbol{q}})$ linearly depends on $\dot{\boldsymbol{q}}$. That condition applies to any robot with only revolute joints, for example.

\section{A. Control task hierarchy and task space definitions}

A control task hierarchy is introduced which comprises $r \in \mathbb{N}$ hierarchy levels. Accordingly, $r$ task-space position coordinates

$$
\boldsymbol{x}_{i}=\boldsymbol{f}_{i}(\boldsymbol{q})
$$

are defined for $i=1 \ldots r$ with the task mapping $\boldsymbol{f}_{i}(\boldsymbol{q}) \in \mathbb{R}^{m_{i}}$ and the corresponding task dimension $m_{i} \in \mathbb{N}$. The following two assumptions are made for the task hierarchy and the workspace in which those tasks are executed.

Assumption 1: All control tasks are structurally feasible at the same time, and the total task dimension matches the number of DOF of the system:

$$
\sum_{i=1}^{r} m_{i}=n .
$$

This assumption will be justified in the discussion in Section V-A.

Assumption 2: The considered workspace is free of singularities.

The restriction on a singularity-free workspace is a common assumption for the analysis of task-space controllers. The implications of Assumption 2 and its justification will also be addressed in Section V-A.

The task space velocities can be written as

$$
\dot{\boldsymbol{x}}_{i}=\boldsymbol{J}_{i}(\boldsymbol{q}) \dot{\boldsymbol{q}}
$$

with the Jacobian matrices $\boldsymbol{J}_{i}(\boldsymbol{q}) \in \mathbb{R}^{m_{i} \times n}$ for all hierarchy levels $i=1 \ldots r$ obtained through

$$
\boldsymbol{J}_{i}(\boldsymbol{q})=\frac{\partial \boldsymbol{f}_{i}(\boldsymbol{q})}{\partial \boldsymbol{q}} .
$$

Furthermore, the so-called augmented Jacobian matrix [3]

$$
\boldsymbol{J}_{i}^{\text {aug }}(\boldsymbol{q})=\left(\begin{array}{c}
\boldsymbol{J}_{1}(\boldsymbol{q}) \\
\vdots \\
\boldsymbol{J}_{i}(\boldsymbol{q})
\end{array}\right)
$$

is introduced which stacks all level-specific Jacobian matrices top-down the task hierarchy down to level $i$. Note that $\boldsymbol{J}_{r}^{\text {aug }}(\boldsymbol{q})$ 
is invertible in the considered workspace due to Assumption 1 and 2. For later use we define the stacked/augmented taskspace velocities and accelerations:

$$
\dot{\boldsymbol{x}}_{i}^{\mathrm{aug}}=\left(\begin{array}{c}
\dot{\boldsymbol{x}}_{1} \\
\vdots \\
\dot{\boldsymbol{x}}_{i}
\end{array}\right), \quad \ddot{\boldsymbol{x}}_{i}^{\mathrm{aug}}=\left(\begin{array}{c}
\ddot{\boldsymbol{x}}_{1} \\
\vdots \\
\ddot{\boldsymbol{x}}_{i}
\end{array}\right)
$$

According to (6)-(7) one can relate the augmented task space velocities (down to level $i$ ) to the joint velocities via

$$
\dot{\boldsymbol{x}}_{i}^{\mathrm{aug}}=\boldsymbol{J}_{i}^{\mathrm{aug}}(\boldsymbol{q}) \dot{\boldsymbol{q}} .
$$

For each objective in the control task hierarchy a nominal trajectory is available. These desired, continuous taskspace positions, velocities, and accelerations are denoted by $\boldsymbol{x}_{i, \mathrm{des}}(t), \dot{\boldsymbol{x}}_{i, \mathrm{des}}(t), \ddot{\boldsymbol{x}}_{i, \mathrm{des}}(t) \in \mathbb{R}^{m_{i}}$ for $i=1 \ldots r$ with time $t$. Accordingly, the errors in the task-space positions $\tilde{\boldsymbol{x}}_{i} \in \mathbb{R}^{m_{i}}$ are defined as

$$
\tilde{\boldsymbol{x}}_{i}=\boldsymbol{x}_{i}(\boldsymbol{q})-\boldsymbol{x}_{i, \mathrm{des}}(t)
$$

for all hierarchy levels $i=1 \ldots r$. Analogously, the task-space errors in the velocities and accelerations are described by $\dot{\tilde{\boldsymbol{x}}}_{i}, \ddot{\tilde{\boldsymbol{x}}}_{i} \in \mathbb{R}^{m_{i}}$. Similar to (7) the superscript ${ }^{\text {aug }}$ can be utilized to stack the desired task-space velocities and accelerations down to level $i$,

$$
\dot{\boldsymbol{x}}_{i, \mathrm{des}}^{\mathrm{aug}}=\left(\begin{array}{c}
\dot{\boldsymbol{x}}_{1, \mathrm{des}} \\
\vdots \\
\dot{\boldsymbol{x}}_{i, \mathrm{des}}
\end{array}\right), \quad \ddot{\boldsymbol{x}}_{i, \mathrm{des}}^{\mathrm{aug}}=\left(\begin{array}{c}
\ddot{\boldsymbol{x}}_{1, \mathrm{des}} \\
\vdots \\
\ddot{\boldsymbol{x}}_{i, \mathrm{des}}
\end{array}\right),
$$

and to stack the corresponding task-space errors

$$
\dot{\tilde{\boldsymbol{x}}}_{i}^{\text {aug }}=\left(\begin{array}{c}
\dot{\tilde{\boldsymbol{x}}}_{1} \\
\vdots \\
\dot{\tilde{\boldsymbol{x}}}_{i}
\end{array}\right), \quad \ddot{\tilde{\boldsymbol{x}}}_{i}^{\mathrm{aug}}=\left(\begin{array}{c}
\ddot{\tilde{\boldsymbol{x}}}_{1} \\
\vdots \\
\ddot{\tilde{\boldsymbol{x}}}_{i}
\end{array}\right) .
$$

\section{B. Dynamically consistent null space projection}

To implement a strict control task hierarchy, dynamically consistent null space projectors $\boldsymbol{N}_{i}(\boldsymbol{q}) \in \mathbb{R}^{n \times n}$ for all levels $i=1 \ldots r$ can be utilized [12], [29]:

$$
\boldsymbol{N}_{i}(\boldsymbol{q})= \begin{cases}\boldsymbol{I} & \text { for } i=1 \\ \boldsymbol{I}-\boldsymbol{J}_{i-1}^{\mathrm{aug}}(\boldsymbol{q})^{T} \boldsymbol{J}_{i-1}^{\mathrm{aug}}(\boldsymbol{q})^{\boldsymbol{M}+, T} & \text { for } i=2 \ldots r\end{cases}
$$

with the identity matrix $I .{ }^{1}$ The operator $M+$ in the superscript indicates the dynamically consistent pseudoinversion of the respective matrix. For more information about the properties of dynamic consistency, the reader is referred to [29].

In order to perform a coordinate transformation of (1) to hierarchically decoupled equations of motion, one has to dynamically decouple the Jacobian matrices (5) to obtain $\overline{\boldsymbol{J}}_{i}(\boldsymbol{q}) \in \mathbb{R}^{m_{i} \times n}$. The straightforward way is to make use of (12) following

$$
\overline{\boldsymbol{J}}_{i}(\boldsymbol{q})=\boldsymbol{J}_{i}(\boldsymbol{q}) \boldsymbol{N}_{i}(\boldsymbol{q})^{T}
$$

\footnotetext{
${ }^{1}$ Note that $\boldsymbol{I}$ is used with different dimensions throughout this work, yet always representing the (square) identity matrix with suitable size.
}

Similar to (4), one obtains new, hierarchically decoupled task space velocities $\boldsymbol{v}_{i} \in \mathbb{R}^{m_{i}}$ :

$$
\underbrace{\left(\begin{array}{c}
\boldsymbol{v}_{1} \\
\vdots \\
\boldsymbol{v}_{r}
\end{array}\right)}_{\boldsymbol{v}}=\underbrace{\left(\begin{array}{c}
\overline{\boldsymbol{J}}_{1}(\boldsymbol{q}) \\
\vdots \\
\overline{\boldsymbol{J}}_{r}(\boldsymbol{q})
\end{array}\right)}_{\overline{\boldsymbol{J}}(\boldsymbol{q})} \dot{\boldsymbol{q}} .
$$

The term $\boldsymbol{v} \in \mathbb{R}^{n}$ stands for the complete task space velocity vector in decoupled coordinates, and $\overline{\boldsymbol{J}}(\boldsymbol{q}) \in \mathbb{R}^{n \times n}$ represents the corresponding, invertible Jacobian matrix.

\section{Hierarchically decoupled equations of motions}

With (14) one can conduct the coordinate transformation

$$
\boldsymbol{\Lambda}(\boldsymbol{q}) \dot{\boldsymbol{v}}+\boldsymbol{\mu}(\boldsymbol{q}, \dot{\boldsymbol{q}}) \boldsymbol{v}=\overline{\boldsymbol{J}}(\boldsymbol{q})^{-T}\left(\boldsymbol{\tau}+\boldsymbol{\tau}^{\mathrm{ext}}-\boldsymbol{g}(\boldsymbol{q})\right)
$$

to obtain hierarchically decoupled equations of motion. The new inertia matrix can be computed through

$$
\begin{aligned}
\boldsymbol{\Lambda}(\boldsymbol{q}) & =\overline{\boldsymbol{J}}(\boldsymbol{q})^{-T} \boldsymbol{M}(\boldsymbol{q}) \overline{\boldsymbol{J}}(\boldsymbol{q})^{-1} \\
& =\operatorname{diag}\left(\boldsymbol{\Lambda}_{1}(\boldsymbol{q}), \ldots, \boldsymbol{\Lambda}_{r}(\boldsymbol{q})\right)
\end{aligned}
$$

and features a block-diagonal structure with the symmetric, positive definite subtask inertia matrices $\boldsymbol{\Lambda}_{i}(\boldsymbol{q}) \in \mathbb{R}^{m_{i} \times m_{i}}$ for $i=1 \ldots r$. This decoupling in the inertial behavior is an intuitive, physical interpretation of dynamic consistency. The Coriolis/centrifugal matrix in (15) can be calculated via

$$
\boldsymbol{\mu}(\boldsymbol{q}, \dot{\boldsymbol{q}})=\overline{\boldsymbol{J}}^{-T}\left(\boldsymbol{C}(\boldsymbol{q}, \dot{\boldsymbol{q}})-\boldsymbol{M}(\boldsymbol{q}) \overline{\boldsymbol{J}}(\boldsymbol{q})^{-1} \dot{\overline{\boldsymbol{J}}}(\boldsymbol{q}, \dot{\boldsymbol{q}})\right) \overline{\boldsymbol{J}}(\boldsymbol{q})^{-1}
$$

and is fully occupied in general. In other words, (15) implies a remaining dynamic coupling between the hierarchy levels due to Coriolis and centrifugal effects.

\section{CONTROL ApProACH}

While the equations of motion (15) feature the beneficial property of inertial decoupling of all $r$ hierarchy levels, the velocities $\boldsymbol{v}$ are rather unintuitive because they are not identical with the original ones (4) anymore. In this section the conversion of the equations of motion back to the original task space will be performed, and the control law will be derived to solve the trajectory tracking problem.

\section{A. Relation between original and hierarchically decoupled task-space velocities}

One can apply (14) and (8) for $i=r$ to relate the original to the new task-space velocities:

$$
\left(\begin{array}{c}
\boldsymbol{v}_{1} \\
\vdots \\
\boldsymbol{v}_{r}
\end{array}\right)=\underbrace{\overline{\boldsymbol{J}}(\boldsymbol{q}) \boldsymbol{J}_{r}^{\mathrm{aug}}(\boldsymbol{q})^{-1}}_{\boldsymbol{B}(\boldsymbol{q})}\left(\begin{array}{c}
\dot{\boldsymbol{x}}_{1} \\
\vdots \\
\dot{\boldsymbol{x}}_{r}
\end{array}\right)
$$

with the lower-triangular matrix $\boldsymbol{B}(\boldsymbol{q}) \in \mathbb{R}^{n \times n}$ containing the submatrices $\boldsymbol{B}_{i, j}(\boldsymbol{q}) \in \mathbb{R}^{m_{i} \times m_{j}}$ with two particular properties: $\boldsymbol{B}_{i, j}(\boldsymbol{q})=\boldsymbol{I}$ for $i=j$, and $\boldsymbol{B}_{i, j}(\boldsymbol{q})=\mathbf{0}$ for $i<j$. The first property describes that the new task-space velocity $\boldsymbol{v}_{i}$ on hierarchy level $i$ involves the original velocity $\dot{\boldsymbol{x}}_{i}$ one-toone, that is, it properly describes the genuine task. The second 
property states that task-space velocities on lower-priority levels do not disturb higher-priority task-space velocities, which ensures the desired strict hierarchy. Accordingly, one can represent all level-specific velocities $\boldsymbol{v}_{i}$ for $i=1 \ldots r$ as functions of the original task-space velocities as follows:

$$
\boldsymbol{v}_{i}=\dot{\boldsymbol{x}}_{i}+\sum_{j=1}^{i-1} \boldsymbol{B}_{i, j}(\boldsymbol{q}) \dot{\boldsymbol{x}}_{j}
$$

Based on (19) one can straightforwardly derive the corresponding task-space accelerations according to

$$
\dot{\boldsymbol{v}}_{i}=\ddot{\boldsymbol{x}}_{i}+\sum_{j=1}^{i-1}\left(\boldsymbol{B}_{i, j}(\boldsymbol{q}) \ddot{\boldsymbol{x}}_{j}+\dot{\boldsymbol{B}}_{i, j}(\boldsymbol{q}, \dot{\boldsymbol{q}}) \dot{\boldsymbol{x}}_{j}\right) .
$$

Due to the dynamic consistency of the null space projections by (12) top-down interferences across the task hierarchy are encountered on velocity- and acceleration level as shown in (19) and (20), respectively.

\section{B. Relation between original and hierarchically decoupled task-space external forces/torques}

The generalized external forces $\boldsymbol{F}_{\dot{\boldsymbol{x}}_{i}}^{\text {ext }} \in \mathbb{R}^{m_{i}}$ in the original task space are collocated to $\dot{\boldsymbol{x}}_{i}$ and can be related to $\boldsymbol{\tau}^{\text {ext }}$ when applying the respective kinematic mapping according to

$$
\boldsymbol{\tau}^{\mathrm{ext}}=\boldsymbol{J}_{r}^{\mathrm{aug}}(\boldsymbol{q})^{T}\left(\begin{array}{c}
\boldsymbol{F}_{\dot{\boldsymbol{x}}_{1}}^{\mathrm{ext}} \\
\vdots \\
\boldsymbol{F}_{\dot{\boldsymbol{x}}_{r}}^{\text {ext }}
\end{array}\right)
$$

Similar to (18), the relation between $\boldsymbol{F}_{\dot{\boldsymbol{x}}_{i}}^{\text {ext }} \in \mathbb{R}^{m_{i}}$ and the corresponding generalized external forces $\boldsymbol{F}_{\boldsymbol{v}_{i}}^{\text {ext }} \in \mathbb{R}^{m_{i}}$ (collocated to $\boldsymbol{v}_{i}$ ) in the decoupled space can be established through

$$
\left(\begin{array}{c}
\boldsymbol{F}_{\boldsymbol{v}_{1}}^{\mathrm{ext}} \\
\vdots \\
\boldsymbol{F}_{\boldsymbol{v}_{r}}^{\mathrm{ext}}
\end{array}\right)=\underbrace{\overline{\boldsymbol{J}}(\boldsymbol{q})^{-T} \boldsymbol{J}_{r}^{\mathrm{aug}}(\boldsymbol{q})^{T}}_{\boldsymbol{E}(\boldsymbol{q})}\left(\begin{array}{c}
\boldsymbol{F}_{\dot{\boldsymbol{x}}_{1}}^{\mathrm{ext}} \\
\vdots \\
\boldsymbol{F}_{\dot{\boldsymbol{x}}_{r}}^{\mathrm{ext}}
\end{array}\right)
$$

with

$$
\boldsymbol{E}(\boldsymbol{q})=\boldsymbol{B}(\boldsymbol{q})^{-T}
$$

In contrast to the lower-triangular structure of $\boldsymbol{B}(\boldsymbol{q})$ the matrix $\boldsymbol{E}(\boldsymbol{q}) \in \mathbb{R}^{n \times n}$ is upper-triangular and contains the submatrices $\boldsymbol{E}_{i, j}(\boldsymbol{q}) \in \mathbb{R}^{m_{i} \times m_{j}}$ with $\boldsymbol{E}_{i, j}(\boldsymbol{q})=\boldsymbol{I}$ for $i=j$. Similar to (19), the generalized external forces from the original taskspace on hierarchy level $i$ enter the decoupled task-space dynamics on level $i$ one-to-one but the cross-coupling is reversed:

$$
\boldsymbol{F}_{\boldsymbol{v}_{i}}^{\mathrm{ext}}=\boldsymbol{F}_{\dot{\boldsymbol{x}}_{i}}^{\mathrm{ext}}+\sum_{j=i+1}^{r} \boldsymbol{E}_{i, j}(\boldsymbol{q}) \boldsymbol{F}_{\dot{\boldsymbol{x}}_{j}}^{\mathrm{ext}}
$$

for $i=1 \ldots r$. In the following the dependencies are omitted in the notations if not strictly necessary for the understanding.

\section{Control law}

The general form of the control law can be written as

$$
\boldsymbol{\tau}=\boldsymbol{g}+\boldsymbol{\tau}_{\boldsymbol{\mu}}+\sum_{i=1}^{r} \boldsymbol{N}_{i} \boldsymbol{J}_{i}^{T} \boldsymbol{F}_{i, \mathrm{ctrl}}
$$

Beside gravity compensation, the compensation of those Coriolis and centrifugal terms is performed which dynamically couple the subtasks [24] as discussed on the basis of (15). Therefore, the respective control action $\tau_{\mu} \in \mathbb{R}^{n}$ annihilates the outer-block-diagonal elements in (17):

$$
\boldsymbol{\tau}_{\boldsymbol{\mu}}=\sum_{i=1}^{r}\left(\overline{\boldsymbol{J}}_{i}^{T}\left(\sum_{j=1}^{i-1} \boldsymbol{\mu}_{i, j} \boldsymbol{v}_{j}+\sum_{j=i+1}^{r} \boldsymbol{\mu}_{i, j} \boldsymbol{v}_{j}\right)\right)
$$

A beneficial peculiarity of (26) is that it constitutes a powerconserving feedback [24]. That means the transmitted power is always zero $\left(\dot{\boldsymbol{q}}^{T} \boldsymbol{\tau}_{\boldsymbol{\mu}}=0\right)$, independent of modeling and parameter uncertainties in $\boldsymbol{\mu}(\boldsymbol{q}, \dot{\boldsymbol{q}})$. This property is due to $\boldsymbol{\mu}_{i, j}=-\boldsymbol{\mu}_{j, i}^{T}$ which, in turn, results from the block-diagonality of $\boldsymbol{\Lambda}(\boldsymbol{q})$. This characteristic is very useful from a robustness point of view and represents a fundamental difference to the non-power-conserving annihilation of all Coriolis and centrifugal terms during the process of feedback linearization in the OSF and classical inverse dynamics.

The last term in (25) is responsible for the task execution on all subtask levels and implements the control forces $\boldsymbol{F}_{i, \mathrm{ctrl}} \in \mathbb{R}^{m_{i}}$. Applying (25) to the system (1) yields

$$
\boldsymbol{\Lambda}_{i} \dot{\boldsymbol{v}}_{i}+\boldsymbol{\mu}_{i, i} \boldsymbol{v}_{i}=\boldsymbol{F}_{i, \mathrm{ctrl}}+\boldsymbol{F}_{\boldsymbol{v}_{i}}^{\mathrm{ext}}
$$

for $i=1 \ldots r$. While (27) basically represents the same structure as in the regulation control problem [24], the main difference lies in the specification of $\boldsymbol{F}_{i, \mathrm{ctrl}}$ as will be shown. By means of (19)-(24) one can go back to the original task space and reformulate (27) to

$$
\boldsymbol{\Lambda}_{i} \ddot{\boldsymbol{x}}_{i}+\boldsymbol{\mu}_{i, i} \dot{\boldsymbol{x}}_{i}+\boldsymbol{\gamma}_{i}(\boldsymbol{q}, \dot{\boldsymbol{q}})\left(\begin{array}{c}
\dot{\boldsymbol{x}}_{i-1}^{\mathrm{aug}} \\
\ddot{\boldsymbol{x}}_{i-1}^{\mathrm{aug}}
\end{array}\right)=\boldsymbol{F}_{i, \mathrm{ctrl}}+\boldsymbol{F}_{\boldsymbol{v}_{i}}^{\mathrm{ext}}
$$

for $i=1 \ldots r$. The term $\gamma_{i}(\boldsymbol{q}, \dot{\boldsymbol{q}}) \in \mathbb{R}^{m_{i} \times 2 \sum_{j=1}^{i-1} m_{j}}$ is defined as

$$
\begin{aligned}
\gamma_{i}(\boldsymbol{q}, \dot{\boldsymbol{q}}) & =\left(\boldsymbol{\Gamma}_{i, 1}, \cdots, \boldsymbol{\Gamma}_{i, i-1}, \boldsymbol{\Psi}_{i, 1}, \cdots, \boldsymbol{\Psi}_{i, i-1}\right) \\
\boldsymbol{\Gamma}_{i, j}(\boldsymbol{q}, \dot{\boldsymbol{q}}) & =\boldsymbol{\mu}_{i, i} \boldsymbol{B}_{i, j}+\boldsymbol{\Lambda}_{i} \dot{\boldsymbol{B}}_{i, j} \\
\boldsymbol{\Psi}_{i, j}(\boldsymbol{q}) & =\boldsymbol{\Lambda}_{i} \boldsymbol{B}_{i, j}
\end{aligned}
$$

where the quantities $\boldsymbol{\Gamma}_{i, j}(\boldsymbol{q}, \dot{\boldsymbol{q}}), \boldsymbol{\Psi}_{i, j}(\boldsymbol{q}) \in \mathbb{R}^{m_{i} \times m_{j}}$ have to be evaluated for $j<i$ only. Multiplying $\gamma_{i}(\boldsymbol{q}, \dot{\boldsymbol{q}})$ by the augmented task-space velocities and accelerations from all higher priority levels $1 \ldots i-1$, as displayed in (28), yields the top-down disturbance across the task hierarchy which affects level $i$.

Based on the equations of motion (28), the control force on hierarchy level $i$ is chosen as

$$
\begin{aligned}
\boldsymbol{F}_{i, \mathrm{ctrl}}= & \boldsymbol{\Lambda}_{i} \ddot{\boldsymbol{x}}_{i, \mathrm{des}}+\boldsymbol{\mu}_{i, i} \dot{\boldsymbol{x}}_{i, \mathrm{des}}-\boldsymbol{D}_{i} \dot{\tilde{\boldsymbol{x}}}_{i}-\boldsymbol{K}_{i} \tilde{\boldsymbol{x}}_{i}+ \\
& +\boldsymbol{\gamma}_{i}(\boldsymbol{q}, \dot{\boldsymbol{q}})\left(\begin{array}{l}
\dot{\boldsymbol{x}}_{i-1, \mathrm{des}}^{\mathrm{aug}} \\
\ddot{\boldsymbol{x}}_{i-1, \mathrm{des}}^{\text {aug }}
\end{array}\right)-\boldsymbol{F}_{i, \mathrm{ctrl}}^{\mathrm{ext}}
\end{aligned}
$$


with the optional annihilation of external forces/torques by

$$
\boldsymbol{F}_{i, \mathrm{ctrl}}^{\mathrm{ext}}= \begin{cases}\mathbf{0} & \text { for case 1 } \\ \sum_{j=i+1}^{r} \boldsymbol{E}_{i, j}(\boldsymbol{q}) \boldsymbol{F}_{\dot{\boldsymbol{x}}_{j}}^{\mathrm{ext}} & \text { for case 2 }\end{cases}
$$

In order to implement the desired impedance, the control force (32) contains the desired, positive definite and symmetric task-space stiffness and damping matrices $\boldsymbol{K}_{i} \in \mathbb{R}^{m_{i} \times m_{i}}$ and $\boldsymbol{D}_{i} \in \mathbb{R}^{m_{i} \times m_{i}}$, respectively. Note that the quantity $\ddot{\boldsymbol{x}}_{i-1, \mathrm{des}}^{\text {aug }} \in \mathbb{R}^{\sum_{1}^{i-1} m_{i}}$ describes a feedforward term based on the known desired trajectory profile, such that it does not contain any measurement or estimation of accelerations.

The choice (33) depends on the desired interaction behavior and the availability of measurements or estimations of interaction forces and torques. The two cases can be distinguished as follows:

- Case 1: This case describes the classical impedance behavior without any feedback of external forces and torques. The main advantage is that one does not require any measuring devices or observers for external disturbances. Cross-couplings with respect to external disturbances can influence the task hierarchy in case of physical interaction with the environment.

- Case 2: This case partially avoids the external force/torque cross-coupling of case 1. Through the topdown disturbances the lower levels can be influenced as will be discussed in Section V. However, if constant external task-space forces/torques are present, these issues do not occur. Knowledge of the interaction forces/torques with the environment is required.

Note that the undisturbed transient behavior of (1) with (25) simplifies to the established hierarchical approach [24] in case of pure regulation control, that is, when the desired trajectories are stopped by setting $\ddot{\boldsymbol{x}}_{i, \mathrm{des}}=\dot{\boldsymbol{x}}_{i, \mathrm{des}}=\mathbf{0}$ on all hierarchy levels $i=1 \ldots r$.

Applying (25) with (26), (32)-(33), to (1) yields

$$
\begin{aligned}
& \boldsymbol{\Lambda}_{i} \ddot{\tilde{\boldsymbol{x}}}_{i}+\left(\boldsymbol{\mu}_{i, i}+\boldsymbol{D}_{i}\right) \dot{\tilde{\boldsymbol{x}}}_{i}+\boldsymbol{K}_{i} \tilde{\boldsymbol{x}}_{i} \\
& =-\gamma_{i}(\boldsymbol{q}, \dot{\boldsymbol{q}})\left(\begin{array}{c}
\dot{\tilde{\boldsymbol{x}}}_{i-1}^{\text {aug }} \\
\ddot{\tilde{\boldsymbol{x}}}_{i-1}^{\text {aug }}
\end{array}\right)+\boldsymbol{F}_{i}^{\text {ext }}
\end{aligned}
$$

for the closed-loop dynamics on hierarchy level $i$, with the resulting, effective interaction

$$
\boldsymbol{F}_{i}^{\text {ext }}= \begin{cases}\boldsymbol{F}_{\boldsymbol{v}_{i}}^{\text {ext }} & \text { for case 1 } \\ \boldsymbol{F}_{\dot{\boldsymbol{x}}_{i}}^{\text {ext }} & \text { for case 2 }\end{cases}
$$

that is characterized by the design choice (33). Note that the top-down disturbance in (34) depends on the higher-priority task-space errors $\dot{\tilde{x}}_{i-1}^{\text {aug }}, \ddot{\tilde{x}}_{i-1}^{\text {aug }}$, see (11).

The closed-loop power flows on hierarchy level $i$ are illustrated in Fig. 2. ${ }^{2}$ The diagram represents the bond graph for (34) and indicates the three ways in which energies can

\footnotetext{
${ }^{2}$ The dotted box in the bond graph in Fig. 2 describes the relation $\dot{\boldsymbol{\Lambda}}_{i}(\boldsymbol{q}, \dot{\boldsymbol{q}})=\boldsymbol{\mu}_{i, i}(\boldsymbol{q}, \dot{\boldsymbol{q}})+\boldsymbol{\mu}_{i, i}(\boldsymbol{q}, \dot{\boldsymbol{q}})^{T}$ through the Dirac structure $\mathcal{D}$ with $\left(\begin{array}{c}\frac{1}{2} \dot{\boldsymbol{\Lambda}}_{i} \dot{\tilde{\boldsymbol{x}}}_{i} \\ -\boldsymbol{\mu}_{i, i} \dot{\tilde{\boldsymbol{x}}}_{i}\end{array}\right)=\left(\begin{array}{cc}\frac{1}{2}\left(\dot{\boldsymbol{\Lambda}}_{i}-2 \boldsymbol{\mu}_{i, i}\right) & \boldsymbol{\mu}_{i, i} \\ -\boldsymbol{\mu}_{i, i}^{T} & -\left(\dot{\boldsymbol{\Lambda}}_{i}-2 \boldsymbol{\mu}_{i, i}^{T}\right)\end{array}\right)\left(\begin{array}{c}\dot{\tilde{\boldsymbol{x}}}_{i} \\ \dot{\tilde{\boldsymbol{x}}}_{i}\end{array}\right)$.
}

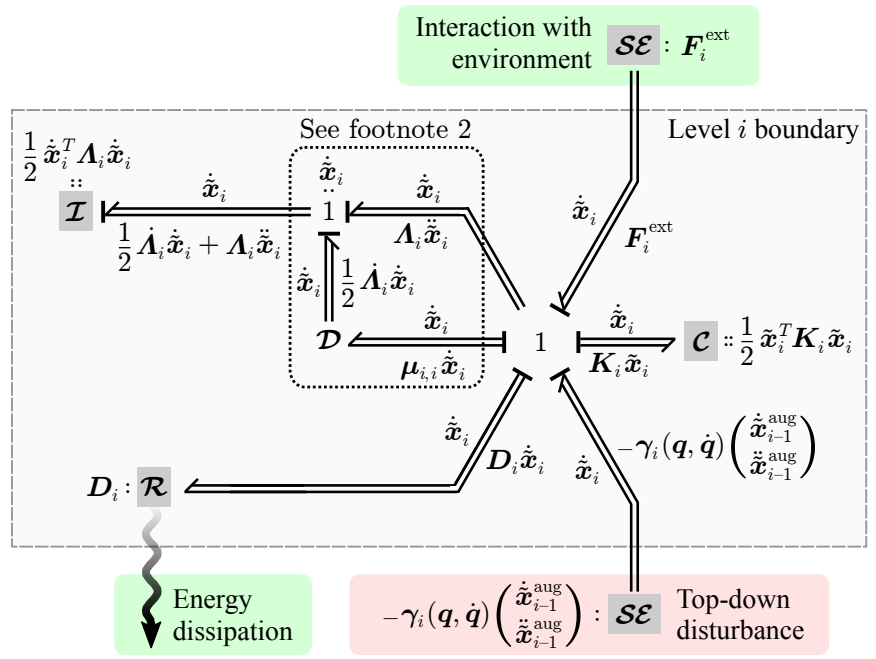

Fig. 2. Bond graph for the power flow on hierarchy level $i$. The depicted boundary encloses the closed-loop dynamics (34). The elements in the bond graph are defined as follows: source of effort $\mathcal{S} \mathcal{E}$, inductance $\mathcal{I}$, capacitance $\mathcal{C}$, resistance $\mathcal{R}$, Dirac structure $\mathcal{D} .^{2}$

cross the level boundary. Through physical interaction via the source of effort $\boldsymbol{F}_{i}^{\text {ext }}$ one can insert or extract energy. For tasks with physical contact this power port describes how the desired impedance characteristics are realized and how external forces and torques influence the energy budget on level $i$. As long as $\dot{\tilde{\boldsymbol{x}}}_{i} \neq \mathbf{0}$, virtual energy is dissipated via the resistance defined by $\boldsymbol{D}_{i}$. The power port at the bottom right originates from the source of effort which describes the topdown disturbance across the hierarchy. Energy can be inserted or extracted through it as long as task-space errors in the velocities or accelerations on the levels $1 \ldots i-1$ are present. When $\ddot{\tilde{\boldsymbol{x}}}_{i-1}^{\text {aug }}=\dot{\tilde{\boldsymbol{x}}}_{i-1}^{\text {aug }}=\mathbf{0}$ this power port vanishes. For more details on bond graph notations, the reader is referred to [30].

\section{STABILITY ANALYSIS}

This section contains the proof of asymptotic stability of the equilibrium in which the task-space tracking errors on all hierarchy levels are zero for the case of free motion $\left(\tau^{\text {ext }}=0\right)$. In this regard the state vectors

$$
\boldsymbol{y}_{i}=\left(\begin{array}{c}
\tilde{\boldsymbol{x}}_{i} \\
\dot{\tilde{\boldsymbol{x}}}_{i}
\end{array}\right), \quad \boldsymbol{y}=\left(\begin{array}{c}
\boldsymbol{y}_{1} \\
\vdots \\
\boldsymbol{y}_{r}
\end{array}\right)
$$

are introduced with $\boldsymbol{y}_{i} \in \mathbb{R}^{2 m_{i}}$ representing the task-space position and velocity error on level $i$ (for $i=1 \ldots r$ ), and $\boldsymbol{y} \in \mathbb{R}^{2 n}$ containing the task-space position and velocity errors of the entire task hierarchy. The above-mentioned equilibrium is consequently defined by $\boldsymbol{y}=\mathbf{0}$. Note that the proof is not valid in the global sense, similar to other stability analyses for task-space controllers where task-space singularities exist. This structural aspect will also be discussed in Section V-A. Moreover, the proof is valid for both cases in (33). As a prerequisite for the following mathematical analysis several quantities have to be introduced in the subsequent Sections IV-A to IV-D. 


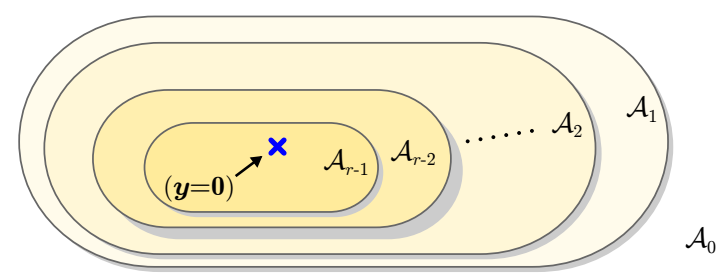

Fig. 3. Nested sets $\mathcal{A}_{0} \ldots \mathcal{A}_{r}$ and the equilibrium $\boldsymbol{y}=\mathbf{0}$ in which all taskspace errors are zero

\section{A. Nested sets for the successive accomplishment of the tasks}

The set $\mathcal{A}_{0}$ describes the complete state space of $\mathbb{R}^{2 n}$ related to (1). In case of free motions one can define the positive invariant set

$$
\mathcal{A}_{1}=\left\{\boldsymbol{y} \mid \dot{\tilde{\boldsymbol{x}}}_{1}=\mathbf{0}, \tilde{\boldsymbol{x}}_{1}=\mathbf{0}\right\}
$$

which describes the fulfillment of the main task. Starting with (37), nested sets $\mathcal{A}_{i}$ for the subordinate hierarchy levels $i=2 \ldots(r-1)$ can be defined in a recursive way. These sets $\mathcal{A}_{2} \ldots \mathcal{A}_{r-1}$ represent the nested fulfillment of the control goals top-down the task hierarchy:

$$
\mathcal{A}_{i}=\mathcal{A}_{i-1} \bigcap\left\{\boldsymbol{y} \mid \dot{\tilde{\boldsymbol{x}}}_{i}=\mathbf{0}, \tilde{\boldsymbol{x}}_{i}=\mathbf{0}\right\}
$$

These nested sets and the equilibrium $\boldsymbol{y}=\mathbf{0}$ are illustrated in Fig. 3.

\section{B. Top-down disturbance across the task hierarchy}

While (34) is always valid one can simplify the equations of motion on level $i$ if the situation is considered where all higher-priority task-space errors are assumed to be zero, i. e., the dynamics are restricted to the corresponding subsets (37) (38). Then the top-down disturbance vanishes:

$$
\gamma_{i}(\boldsymbol{q}, \dot{\boldsymbol{q}})\left(\begin{array}{c}
\dot{\tilde{\boldsymbol{x}}}_{i-1}^{\mathrm{aug}} \\
\ddot{\tilde{\boldsymbol{x}}}_{i-1}^{\text {aug }}
\end{array}\right)=\mathbf{0} \quad \forall \boldsymbol{y} \in \mathcal{A}_{i-1}
$$

and the equations of motion reduce to

$$
\boldsymbol{\Lambda}_{i} \ddot{\tilde{\boldsymbol{x}}}_{i}+\left(\boldsymbol{\mu}_{i, i}+\boldsymbol{D}_{i}\right) \dot{\tilde{\boldsymbol{x}}}_{i}+\boldsymbol{K}_{i} \tilde{\boldsymbol{x}}_{i}=\boldsymbol{F}_{i}^{\mathrm{ext}} \forall \boldsymbol{y} \in \mathcal{A}_{i-1}
$$

\section{Level-specific energies and powers}

For the case of free motion an energy- and power-like function on level $i$ related to (40) can be written as

$$
\begin{array}{rlrl}
V_{i} & =\frac{1}{2} \boldsymbol{y}_{i}^{T}\left(\begin{array}{cc}
\boldsymbol{K}_{i} & \mathbf{0} \\
\mathbf{0} & \boldsymbol{\Lambda}_{i}
\end{array}\right) \boldsymbol{y}_{i} & & \forall \boldsymbol{y} \in \mathcal{A}_{i-1} \\
\dot{V}_{i} & =-\boldsymbol{y}_{i}^{T}\left(\begin{array}{cc}
\mathbf{0} & \mathbf{0} \\
\mathbf{0} & \boldsymbol{D}_{i}
\end{array}\right) \boldsymbol{y}_{i} & \forall \boldsymbol{y} \in \mathcal{A}_{i-1}
\end{array}
$$

containing the spring potential and a velocity-error-related part on this very level. Note that the time derivative of the inertia matrix $\dot{\Lambda}_{i}$ in (42) vanishes due to $\dot{\Lambda}_{i}=\boldsymbol{\mu}_{i, i}+\boldsymbol{\mu}_{i, i}^{T}$. Obviously,
$V_{i}$ is positive definite in $\boldsymbol{y}_{i}$, whereas $\dot{V}_{i}$ is only negative semidefinite in $\boldsymbol{y}_{i}$ for $i=1 \ldots r$. The following modified versions of (41)-(42) are introduced as

$$
\begin{aligned}
V_{i, \epsilon} & =\frac{1}{2} \boldsymbol{y}_{i}^{T} \underbrace{\left(\begin{array}{cc}
\boldsymbol{K}_{i} & \epsilon_{i} \boldsymbol{\Lambda}_{i} \\
\epsilon_{i} \boldsymbol{\Lambda}_{i}^{T} & \boldsymbol{\Lambda}_{i}
\end{array}\right)}_{\boldsymbol{P}_{i}^{\text {ene }}} \boldsymbol{y}_{i} \\
\dot{V}_{i, \epsilon} & =-\boldsymbol{y}_{i}^{T} \underbrace{\left(\begin{array}{cc}
\epsilon_{i} \boldsymbol{K}_{i} \\
\frac{1}{2} \epsilon_{i}\left(\boldsymbol{D}_{i}-\boldsymbol{\mu}_{i, i}^{T}\right)^{T} & \frac{1}{2} \epsilon_{i}\left(\boldsymbol{D}_{i}-\boldsymbol{\mu}_{i, i}^{T}\right) \\
\boldsymbol{D}_{i}-\epsilon_{i} \boldsymbol{\Lambda}_{i}
\end{array}\right)}_{\boldsymbol{P}_{i}^{\text {pow }}} \boldsymbol{y}_{i}
\end{aligned}
$$

$\forall \boldsymbol{y} \in \mathcal{A}_{i-1}$ by skewing the level sets of the energy-like function via a state-coupling term based on the parameter $\epsilon_{i}>0$ [28], [31]. For sufficiently small parameters $\epsilon_{i}$, one can state that $V_{i, \epsilon}$ is positive definite in $\boldsymbol{y}_{i}$, that is $\boldsymbol{P}_{i}^{\text {ene }} \succ 0$, since $\boldsymbol{\Lambda}_{i}, \boldsymbol{K}_{i} \succ 0$. Moreover, $\dot{V}_{i, \epsilon}$ is negative definite for sufficiently small values of $\epsilon_{i}$. That can be shown by resorting to the Schur complement conditions [32] for the positive definiteness of $\boldsymbol{P}_{i}^{\text {pow }}$. Since $\boldsymbol{K}_{i}, \boldsymbol{D}_{i}, \boldsymbol{\Lambda}_{i}$ are symmetric and positive definite, $\boldsymbol{P}_{i}^{\text {pow }} \succ 0$ can be stated iff

$$
\begin{aligned}
\epsilon_{i} \boldsymbol{K}_{i} & \succ 0 \\
\boldsymbol{D}_{i}-\epsilon_{i}\left(\boldsymbol{\Lambda}_{i}+\frac{1}{4}\left(\boldsymbol{D}_{i}-\boldsymbol{\mu}_{i, i}^{T}\right)^{T} \boldsymbol{K}_{i}^{-1}\left(\boldsymbol{D}_{i}-\boldsymbol{\mu}_{i, i}^{T}\right)\right) & \succ 0
\end{aligned}
$$

holds true. Note that the term on the left of (46) is the Schur complement of $\epsilon_{i} \boldsymbol{K}_{i}$. Since $\boldsymbol{K}_{i}, \boldsymbol{D}_{i}, \boldsymbol{\Lambda}_{i}$ are known to be lower- and upper-bound, satisfying (46) for sufficiently small parameters $\epsilon_{i}$ only depends on the boundedness of $\boldsymbol{\mu}_{i, i}(\boldsymbol{q}, \dot{\boldsymbol{q}})$ or $\dot{\boldsymbol{q}}$, respectively. The boundedness of $\dot{\boldsymbol{q}}$ and (consequently the boundedness of $\boldsymbol{\mu}_{i, i}(\boldsymbol{q}, \dot{\boldsymbol{q}})$ ) will be shown in Section IV-E by the use of a theorem which makes it possible to conclude stability, i. e., boundedness of the states $\dot{\boldsymbol{q}}$.

Summarized, one can find functions $V_{i, \epsilon}, \dot{V}_{i, \epsilon}$ with the properties $V_{i, \epsilon} \succ 0$ and $\dot{V}_{i, \epsilon} \prec 0$ within the subset $\mathcal{A}_{i-1}$.

\section{Theorems on uniform (asymptotic) stability}

For the line of argumentation in the proof in Section IV-E two theorems from the field of stability theory are used which, in turn, require an additional (straightforward) assumption.

Consider the time-varying system

$$
\dot{\boldsymbol{z}}=\boldsymbol{h}(t, \boldsymbol{z}), \quad \boldsymbol{z}\left(t_{0}\right)=\boldsymbol{z}_{0},
$$

where $\boldsymbol{h}: \mathbb{R}_{\geq 0} \times \mathcal{D} \rightarrow \mathbb{R}^{n}, \mathcal{D} \subset \mathbb{R}^{n}$ is a domain with $\boldsymbol{z}=\mathbf{0} \in \mathcal{D}$ and $\boldsymbol{h}(t, \mathbf{0}) \equiv \mathbf{0}$ for all $t \geq t_{0}$. The solution of (47) at any time instant $t$ is denoted as $\boldsymbol{z}(t)$ [26]. The function $\boldsymbol{h}(t, \boldsymbol{z})$ is continuous and locally Lipschitz in $\boldsymbol{z}$ uniformly in $t$, i. e., for any compact set $\mathcal{B} \subset \mathcal{D}$, there exists a constant $L_{\mathcal{B}}>0$, independent of $t$, such that

$$
\left\|\boldsymbol{h}\left(t, \boldsymbol{z}_{1}\right)-\boldsymbol{h}\left(t, \boldsymbol{z}_{2}\right)\right\| \leq L_{\mathcal{B}}\left\|\boldsymbol{z}_{1}-\boldsymbol{z}_{2}\right\|
$$

for any $\boldsymbol{z}_{1}, \boldsymbol{z}_{1} \in \mathcal{B}$ and $t \in \mathbb{R}$. Note that these properties are widely used to ensure existence and uniqueness of the solutions of time-varying systems [26], [33]. Therefore, we state the following assumption for the robotic system at hand:

Assumption 3: The closed-loop system described by (34) fulfills the same requirements as (47) concerning existence and uniqueness of the solutions. 


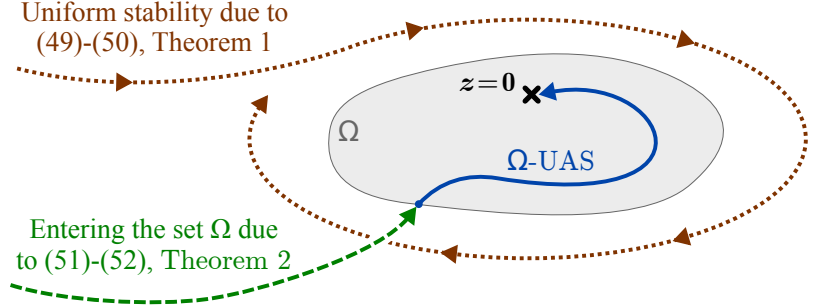

Fig. 4. Interpretation of the Theorems 1 and 2. The conditional uniform asymptotic stability of $\boldsymbol{z}=\mathbf{0}$, that is, $\Omega$-UAS, is exemplified by the solid/blue trajectory. The stability in Theorem 1 is due to the negative semi-definiteness in (50), see the dotted/brown curve. The asymptotic stability in Theorem 2 is due to the negative definiteness in (52), see the dashed/green line.

Furthermore, several definitions from stability theory [26], [33], [34] will be used in the proof: uniform stability (US), uniform asymptotic stability (UAS), and these definitions conditionally to subsets $\mathcal{Z}$, i. e., $\mathcal{Z}$-US and $\mathcal{Z}$-UAS. The detailed descriptions and properties can be found in Appendix A. With these prerequisites the following two theorems can be applied.

Theorem 1: [26] Suppose that Assumption 3 holds for (47). If there exists a function $V(t, \boldsymbol{z}) \in \mathcal{C}^{1}\left(\mathbb{R}_{\geq t_{0}} \times \mathcal{D}, \mathbb{R}_{\geq 0}\right)$ with positive semi-definite functions $W_{j}(\boldsymbol{z}), j=1,2$, such that

$$
\begin{aligned}
& W_{1}(\boldsymbol{z}) \leq V(t, \boldsymbol{z}) \leq W_{2}(\boldsymbol{z}) \\
& \frac{\partial V}{\partial t}+\left(\frac{\partial V}{\partial \boldsymbol{z}}\right)^{T} \boldsymbol{h}(t, \boldsymbol{z}) \leq 0
\end{aligned}
$$

for all $t \geq 0$ and $\boldsymbol{z} \in \mathcal{D}$, then the equilibrium $\boldsymbol{z}=\mathbf{0}$ of (47) is US if it is $\Omega$-UAS, where $\Omega \triangleq\left\{\boldsymbol{z} \in \mathcal{D} \mid W_{1}(\boldsymbol{z})=0\right\}$.

The proof of Theorem 1 is provided in [26].

Theorem 2: [26] Suppose that Assumption 3 holds for the system (47). Assume that there exists a function $V(t, \boldsymbol{z}) \in \mathcal{C}^{1}\left(\mathbb{R}_{\geq t_{0}} \times \mathcal{D}, \mathbb{R}_{\geq 0}\right)$ with positive semi-definite functions $W_{j}(\boldsymbol{z}), j=1,2$, such that

$$
\begin{aligned}
& W_{1}(\boldsymbol{z}) \leq V(t, \boldsymbol{z}) \leq W_{2}(\boldsymbol{z}) \\
& \frac{\partial V}{\partial t}+\left(\frac{\partial V}{\partial \boldsymbol{z}}\right)^{T} \boldsymbol{h}(t, \boldsymbol{z}) \leq-\alpha\left(W_{1}(\boldsymbol{z})\right)
\end{aligned}
$$

are satisfied for all $t \geq 0$ and $z \in \mathcal{D}$ and $\alpha \in \mathcal{K}^{3}$ Then the equilibrium $\boldsymbol{z}=\mathbf{0}$ of (47) is UAS if it is $\Omega$-UAS, where $\Omega \triangleq\left\{\boldsymbol{z} \in \mathcal{D} \mid W_{1}(\boldsymbol{z})=0\right\}$.

The proof of Theorem 2 is provided in [26].

The main difference between Theorem 1 and 2 lies in the right side of the equations (50) (negative semi-definiteness) and (52) (negative definiteness), respectively. An interpretation of both theorems is provided in Fig. 4.

In Section IV-E, these two theorems will be applied on the individual hierarchy levels of the closed-loop system (34) with $V(t, \boldsymbol{z})$ taken from (41) for Theorem 1 and taken from (43) for Theorem 2, while the sets $\Omega$ are selected according to the nested, task-related sets in Section IV-A. The lower and upper bounds $W_{1}(\boldsymbol{z})$ and $W_{2}(\boldsymbol{z})$ can be derived from $V(t, \boldsymbol{z})$ by means of minimal and maximal eigenvalues.

\footnotetext{
${ }^{3} \mathrm{~A}$ function of class $\mathcal{K}$ is continuous, zero at zero, and strictly increasing.
}

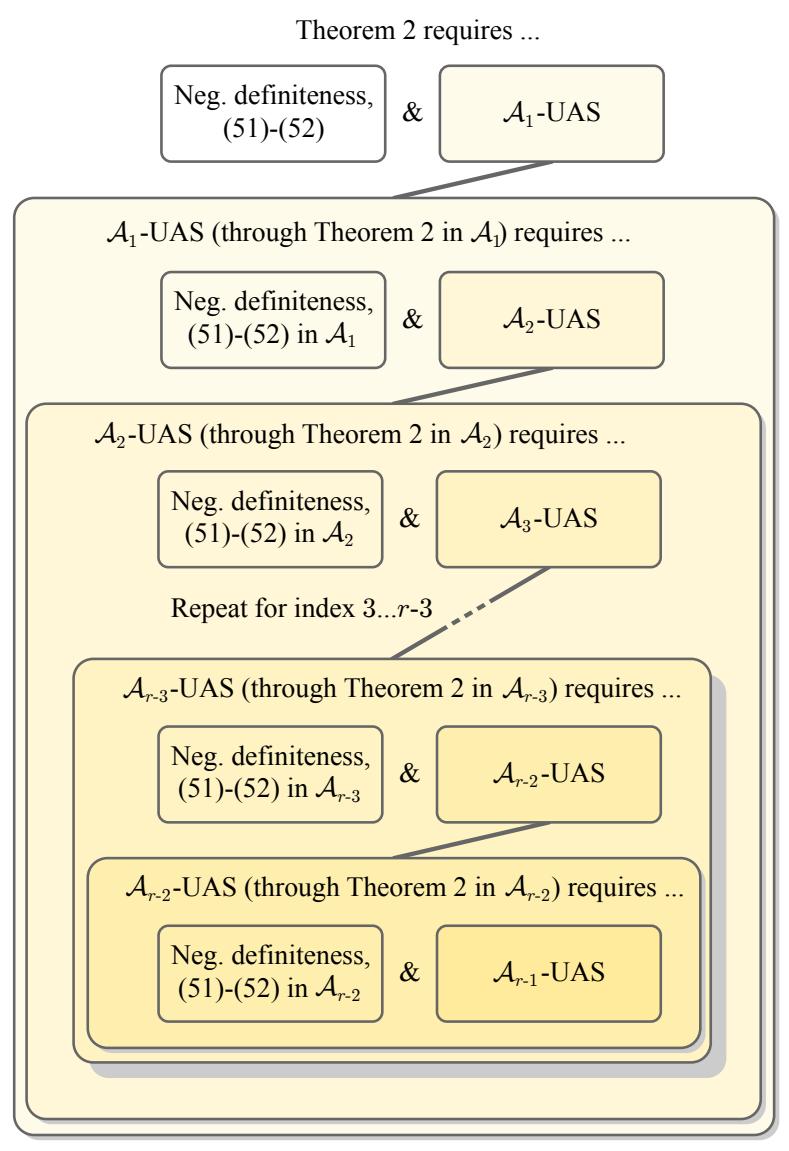

Fig. 5. Structure of the proof of asymptotic stability of the equilibrium $\boldsymbol{y}=\mathbf{0}$

\section{E. Proof of asymptotic stability}

Proposition 1: Consider the system (1) with the control law (25). The stiffness and damping matrices $\boldsymbol{K}_{i}, \boldsymbol{D}_{i}$ for $i=1 \ldots r$ are symmetric and positive definite, respectively, and the desired task-space positions, velocities, and accelerations $\left(\boldsymbol{x}_{i, \mathrm{des}}(t), \dot{\boldsymbol{x}}_{i, \mathrm{des}}(t), \ddot{\boldsymbol{x}}_{i, \mathrm{des}}(t)\right.$ for $\left.i=1 \ldots r\right)$ are continuous and bounded. Suppose that the Assumptions 1-3 hold. Then the equilibrium $\boldsymbol{y}=\mathbf{0}$ is asymptotically stable for the case of free motion $\left(\tau^{\mathrm{ext}}=\mathbf{0}\right)$.

Proof 1: The structure of the proof of asymptotic stability is illustrated in Fig. 5. In order to obtain UAS of the equilibrium $\boldsymbol{y}=\mathbf{0}$, one can apply Theorem 2 to the complete closedloop dynamics of the system. Theorem 2 requires functions to satisfy (51)-(52), and it additionally demands $\mathcal{A}_{1}$-UAS. This property $\mathcal{A}_{1}$-UAS can be straightforwardly obtained by applying Theorem 2 within the subset $\mathcal{A}_{1}$. In consequence, one requires functions to satisfy the negative definiteness defined in (51)-(52) within $\mathcal{A}_{1}$, and $\mathcal{A}_{2}$-UAS is additionally demanded. The latter requirement, $\mathcal{A}_{2}$-UAS, in turn, can be obtained by repeating the previous step with increasing index, i. e., topdown the task hierarchy. That procedure can be iteratively applied until $\mathcal{A}_{r-1}$-UAS is finally demanded. Summarized, asymptotic stability of $\boldsymbol{y}=\mathbf{0}$ is proven if one can satisfy all requirements depicted in Fig. 5.

In the following, these requirements will be fulfilled by proving them from bottom to top. In Fig. 6 the procedure 
for the line of argumentation is detailed by the consecutive steps (1) to (7) which realize the structure in Fig. 5.

\section{Step (1), positive definiteness of $\boldsymbol{P}_{r}^{\text {pow }}$ within $\mathcal{A}_{r-1}$}

The line of argumentation starts on the lowest level $i=r$ and within the subset $\mathcal{A}_{r-1}$. Within this subset, one can evaluate (41)-(42) and conclude $\mathcal{A}_{r-1}$-US of the equilibrium. From that one can argue that $\tilde{\boldsymbol{x}}_{r}$ and $\dot{\tilde{\boldsymbol{x}}}_{r}$ are bounded. Since $\tilde{\boldsymbol{x}}_{j}=\dot{\tilde{\boldsymbol{x}}}_{j}=\mathbf{0}$ for $1 \leq j \leq(r-1)$ due to the restriction to $\mathcal{A}_{r-1}$, boundedness of all task-space velocities $\dot{\boldsymbol{x}}_{r}^{\text {aug }}$ can be concluded. With (8) for $i=r$ one can prove boundedness of $\dot{\boldsymbol{q}}$, and therefore, boundedness of $\boldsymbol{\mu}_{r, r}(\boldsymbol{q}, \dot{\boldsymbol{q}})$. That, in turn, confirms $\boldsymbol{P}_{r}^{\text {pow }} \succ 0$ within $\mathcal{A}_{r-1}$, because (45)-(46) is satisfied.

\section{Step (2), $\mathcal{A}_{r-1}-U A S$ of the equilibrium}

The positive definiteness of $\boldsymbol{P}_{r}^{\text {pow }}$ shown in step (1) makes it possible to conclude $\mathcal{A}_{r-1}$-UAS of the equilibrium from (43)-(44) for $i=r$ because $V_{r, \epsilon} \succ 0$ and $\dot{V}_{r, \epsilon} \prec 0$ within the subset $\mathcal{A}_{r-1}$.

Step (3), positive definiteness of $\boldsymbol{P}_{r-1}^{\text {pow }}$ within $\mathcal{A}_{r-2}$

Going one level higher $(i=r-1)$ but restricting all following statements to the subset $\mathcal{A}_{r-2}$, Theorem 1 can be applied with $V \triangleq V_{r-1}$ from (41) and $\Omega \triangleq \mathcal{A}_{r-1} \cdot{ }^{4}$ That yields $\mathcal{A}_{r-2}$ US of the equilibrium. With $\mathcal{A}_{r-2}$-US of the equilibrium one can conclude boundedness of all velocities, and therefore boundedness of $\boldsymbol{\mu}_{r-1, r-1}(\boldsymbol{q}, \dot{\boldsymbol{q}})$ in $\mathcal{A}_{r-2}$. As a consequence, $\boldsymbol{P}_{r-1}^{\text {pow }} \succ 0$ within the subset $\mathcal{A}_{r-2}$ can be stated, because (45)(46) is satisfied.

\section{Step (4), $\mathcal{A}_{r-2}$-UAS of the equilibrium}

Theorem 2 can be applied within $\mathcal{A}_{r-2}$ to obtain $\mathcal{A}_{r-2}$-UAS of the equilibrium. The substitutions $V \triangleq V_{r-1, \epsilon}, \Omega \triangleq \mathcal{A}_{r-1}$, $W_{1} \triangleq W_{r-1,1}$, and $W_{2} \triangleq W_{r-1,2}$ are chosen with

$$
\begin{aligned}
W_{r-1,1} & =\frac{1}{2} \lambda_{\min }\left(\boldsymbol{P}_{r-1}^{\mathrm{ene}}\right)\left\|\boldsymbol{y}_{r-1}\right\|^{2}, \\
W_{r-1,2} & =\frac{1}{2} \lambda_{\max }\left(\boldsymbol{P}_{r-1}^{\mathrm{ene}}\right)\left\|\boldsymbol{y}_{r-1}\right\|^{2} .
\end{aligned}
$$

Herein $\lambda_{\min }$ and $\lambda_{\max }$ define the minimal and maximal eigenvalue of the respective argument. With $\alpha \triangleq \alpha_{r-1}$ in Theorem 2 according to

$$
\alpha_{r-1}=\frac{2 \lambda_{\min }\left(\boldsymbol{P}_{r-1}^{\mathrm{pow}}\right)}{\lambda_{\min }\left(\boldsymbol{P}_{r-1}^{\mathrm{ene}}\right)} W_{r-1,1}
$$

one can ensure that (52) is satisfied since

$$
\dot{V}_{r-1, \epsilon} \leq-\alpha_{r-1}=-\lambda_{\min }\left(\boldsymbol{P}_{r-1}^{\text {pow }}\right)\left\|\boldsymbol{y}_{r-1}\right\|^{2}
$$

That proves $\mathcal{A}_{r-2}$-UAS of the equilibrium.

\footnotetext{
${ }^{4}$ Note that $W_{1}$ and $W_{2}$ can be determined by involving the minimal and maximal eigenvalues obtained from (41) for $i=r-1$, for example.
}

Step (5), iterative application of (3) and (4) bottom-up the task hierarchy, $\mathcal{A}_{1}$-UAS of the equilibrium

The previous two steps can be iteratively repeated, i.e., showing $\boldsymbol{P}_{i}^{\text {pow }} \succ 0$ and proving $\mathcal{A}_{i-1}$-UAS of the equilibrium for the indices $i=(r-2) \ldots 2$. This procedure includes the following four substeps.

Starting index $i$ : For the first iteration, $i$ is set to $r-2$.

Repetition of substep (3): Similar to step (3), Theorem 1 is applied within the subset $\mathcal{A}_{i-1}$ and requires $V \triangleq V_{i}$ and $\Omega \triangleq \mathcal{A}_{i}$. The positive semi-definite functions $W_{1}$ and $W_{2}$ can be determined by involving the minimal and maximal eigenvalues obtained from (41), for example. From Theorem 1 one can conclude boundedness of $\boldsymbol{\mu}_{i, i}(\boldsymbol{q}, \dot{\boldsymbol{q}})$ within the respective subset, and consequently $\boldsymbol{P}_{i}^{\text {pow }} \succ 0$ conditional to $\mathcal{A}_{i-1}$, due to the fulfillment of (45)-(46).

Repetition of substep (4): Analogously, Theorem 2 is applied within the subset $\mathcal{A}_{i-1}$. One can set $V \triangleq V_{i, \epsilon}, \Omega \triangleq \mathcal{A}_{i}$, $W_{1} \triangleq W_{i, 1}, W_{2} \triangleq W_{i, 2}$, and $\alpha \triangleq \alpha_{i}$ with the respective indices replaced in the general formulations

$$
\begin{aligned}
W_{i, 1}\left(\boldsymbol{y}_{i}\right) & =\frac{1}{2} \lambda_{\min }\left(\boldsymbol{P}_{i}^{\mathrm{ene}}\right)\left\|\boldsymbol{y}_{i}\right\|^{2}, \\
W_{i, 2}\left(\boldsymbol{y}_{i}\right) & =\frac{1}{2} \lambda_{\max }\left(\boldsymbol{P}_{i}^{\mathrm{ene}}\right)\left\|\boldsymbol{y}_{i}\right\|^{2}, \\
\alpha_{i}\left(W_{i, 1}\left(\boldsymbol{y}_{i}\right)\right) & =\frac{2 \lambda_{\min }\left(\boldsymbol{P}_{i}^{\mathrm{pow}}\right)}{\lambda_{\min }\left(\boldsymbol{P}_{i}^{\mathrm{ene}}\right)} W_{i, 1}\left(\boldsymbol{y}_{i}\right) .
\end{aligned}
$$

One can conclude $\mathcal{A}_{i-1}$-UAS of the equilibrium.

Update of the index $i$ : The index is updated according to the sequence $i=(r-2) \ldots 2$, and the next iteration is initiated. After $r-3$ iterations, i. e., when $i=2$ is reached, $\mathcal{A}_{1}$-UAS of the equilibrium is proven.

\section{Step (6), positive definiteness of $\boldsymbol{P}_{1}^{\text {pow }}$}

Applying Theorem 1 one last time with $V \triangleq V_{1}$ and $\Omega \triangleq \mathcal{A}_{1}$, one can conclude $\boldsymbol{P}_{1}^{\text {pow }} \succ 0$. The functions $W_{1}$ and $W_{2}$ can be determined analogously as in the previous steps.

\section{Step (7), UAS of the equilibrium}

Applying Theorem 2 one last time with $V \triangleq V_{1, \epsilon}, \Omega \triangleq \mathcal{A}_{1}$, and (57)-(59) for $i=1$ delivers the proof of UAS of the equilibrium $\boldsymbol{y}=\mathbf{0}$.

\section{Discussion}

The proposed controller will be discussed (Section V-A) and compared with the classical approach based on feedback linearization (Section V-B). The conclusions of this comparison are generalizable to other control approaches which are characterized by a feedback linearization such as the classical OSF and common inverse-dynamics solutions [14].

\section{A. General discussion}

Structurally (25)-(33) describes a so-called compliance controller which is as a special case of impedance control because it does not shape the inertia but it preserves the configurationdependent, natural one. In [17] different operational space 


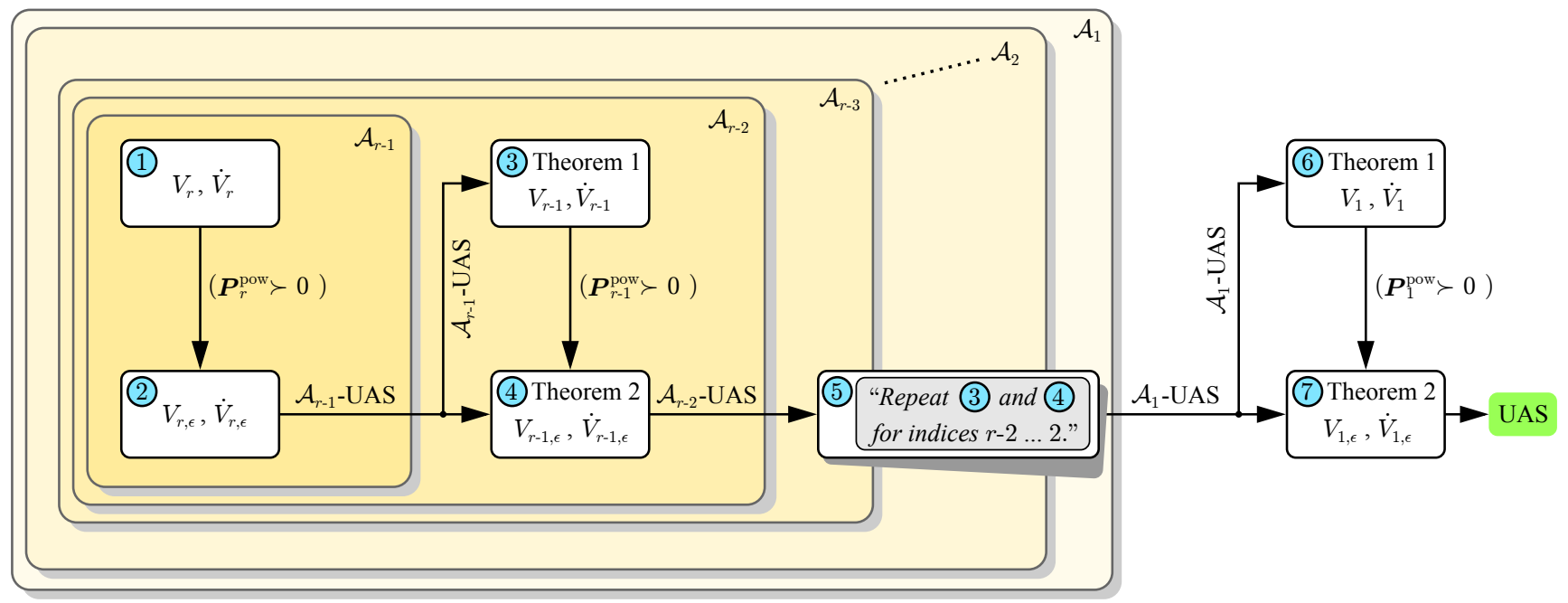

Fig. 6. Detailed procedure to fulfill the requirements of the proof sketched in Fig. 5. The proof of asymptotic stability of $\boldsymbol{y}=\mathbf{0}$ can be performed by following the consecutive steps (1) to (7), where step (5) consists of the iterative application of the steps (3) and (4) with changing index (bottom-up the task hierarchy). After the final application of Theorem 2 in step (7) one can conclude UAS of the equilibrium, i. e., all desired subtask trajectories are tracked.

controllers have been investigated and compared from a theoretical and empirical perspective. The authors came to the conclusion that a highly accurate estimation of the inertia matrix is difficult to obtain. While both feedback linearization and the controller proposed here require knowledge of the inertia matrix, feedback linearization is in general rather prone to modeling uncertainties in $\boldsymbol{M}(\boldsymbol{q})$ since the natural inertia is actively shaped to become constant and preferably diagonal.

Null space projections make it possible to partially execute subordinate tasks if they are in structural conflict with higherpriority objectives, i. e., if the tasks are not consistent with each other or dependent, respectively. While a stability analysis can still be conducted for specific cases (compliance control [35], inverse-dynamics/OSF approach [36]) the proofs are usually only valid for the regulation case and local minima. In general, zero tracking error cannot be achieved on all hierarchy levels in case of conflicting tasks, and the question of existence of an equilibrium is not trivial. The difficulties in analyzing the stability properties have been investigated in [17].

In contrast, the requirements at hand aim at a fully feasible task hierarchy or independent subtasks, respectively. The task planner and high-level reasoning layer are assumed to define the kinematic and dynamic control objectives in a consistent way, which is a common requirement [11]. As a consequence, the presented hierarchical impedance controller is responsible for a) tracking the desired trajectories, b) strictly complying with the order of priority, and c) implementing the desired contact behavior (stiffness, damping). If the desired subtask trajectories are properly planned, both algorithmic and kinematic singularities do not have to be treated in the control approach separately. As in all projection-based approaches, singularities can lead to discontinuities in the control action and have to be addressed otherwise, for example through damped least-squares techniques [37] applied to the control law [38], [39] or smoothing the transition itself [40].

Another aspect to be emphasized is the general structure of the control law (25)-(33). As mentioned in Section III-C, the tracking controller reduces to the regulation controller [24] when removing the time dependencies in the references on all hierarchy levels by setting $\ddot{\boldsymbol{x}}_{i, \mathrm{des}}=\dot{\boldsymbol{x}}_{i, \mathrm{des}}=\mathbf{0}$ for $i=1 \ldots r$. This compliance controller is enhanced here by upgrading it to a dedicated tracking controller while preserving the beneficial interaction properties of an impedance controller [25].

In general, proofs of stability for task-space controllers are not valid in the global sense if singularities exist due to the definition of the subtasks. The same limitation applies, for example, to any Cartesian orientation controller involving all three rotations in $\mathrm{SO}(3)$. This structural property is valid regardless of the type of controller. Although this mathematical restriction has to be noted, its implications in practice are rather limited, when the nominal task-space trajectories are properly planned to avoid singular regions and ensure feasibility of the subtasks [11].

If the cross-couplings with respect to the external forces and torques in (24) are annihilated as described by case 2 in (33), then one can also prove asymptotic stability of the equilibrium in the presence of constant external forces and torques $\boldsymbol{F}_{\dot{\boldsymbol{x}}_{i}}^{\text {ext }} \neq \mathbf{0}$. Such external disturbances can be present on several hierarchy levels simultaneously without loss of generality. The new equilibrium $\boldsymbol{y}$ straightforwardly derives from the corresponding desired task-space stiffnesses and can be shown to be asymptotically stable by following a similar line of argumentation as done in Section IV-E.

\section{B. Comparison with feedback linearization}

In the following the control approach is qualitatively compared with the state of the art, i. e., feedback linearization with the level-specific decoupled equations of motion

$$
\boldsymbol{\Lambda}_{i, \mathrm{des}} \ddot{\tilde{\boldsymbol{x}}}_{i}+\boldsymbol{D}_{i} \dot{\tilde{\boldsymbol{x}}}_{i}+\boldsymbol{K}_{i} \tilde{\boldsymbol{x}}_{i}=\boldsymbol{F}_{\dot{\boldsymbol{x}}_{i}}^{\mathrm{ext}}
$$

of the closed loop for all levels $i=1 \ldots r$. The term $\boldsymbol{\Lambda}_{i, \mathrm{des}} \in \mathbb{R}^{m_{i} \times m_{i}}$ describes the constant, desired task-space inertia matrix. 
1) Advantages over classical feedback linearization: When performing a feedback linearization, the control law requires knowledge of the generalized external forces $\boldsymbol{\tau}^{\text {ext }}$ or $\boldsymbol{F}_{\dot{\boldsymbol{x}}_{i}}^{\text {ext }}$ for $i=1 \ldots r$, respectively. If this feedback is not provided, then the impedance parameters (e.g., the desired stiffness matrices $\boldsymbol{K}_{i}$ for $i=1 \ldots r$ ) will not be implemented on the robot. Physically interacting with the closed-loop system will then lead to a wrong, configuration-dependent contact stiffness because the external force is unintuitively distorted by the factor $\boldsymbol{\Lambda}_{i, \mathrm{des}} \boldsymbol{\Lambda}_{i}^{-1}$. In the approach proposed here, such a distortion does not happen since $\boldsymbol{\Lambda}_{i, \mathrm{des}} \boldsymbol{\Lambda}_{i}^{-1}=\boldsymbol{I}$ due to the preservation of the natural inertia $\left(\boldsymbol{\Lambda}_{i, \mathrm{des}}=\boldsymbol{\Lambda}_{i}\right)$. Moreover, it is well known that the identification of $\tau^{\text {ext }}$, either by measurement of estimation, potentially involves problems in terms of robustness and accuracy. In the approach presented here, feedback of $\tau^{\text {ext }}$ can be completely avoided (case 1 in (33)).

Let the system involve unmodeled friction, for example. This dissipative effect could be taken into account by introducing an unknown/unmodeled velocity-dependent term $\boldsymbol{D}_{i}^{\text {fric }} \dot{\boldsymbol{x}}_{i}$ with positive (semi-)definite damping matrix $\boldsymbol{D}_{i}^{\text {fric }}$. Such a term would appear in (60) as $\boldsymbol{\Lambda}_{i, \mathrm{des}} \boldsymbol{\Lambda}_{i}^{-1} \boldsymbol{D}_{i}^{\text {fric }} \dot{\boldsymbol{x}}_{i}$ due to the previously performed feedback linearization. Although $\boldsymbol{\Lambda}_{i \text {,des }} \succ 0$ and $\boldsymbol{\Lambda}_{i}^{-1} \succ 0$ and $\boldsymbol{D}_{i}^{\text {fric }} \succeq 0$, the term $\boldsymbol{\Lambda}_{i, \mathrm{des}} \boldsymbol{\Lambda}_{i}^{-1} \boldsymbol{D}_{i}^{\text {fric }}$ is not necessarily positive (semi-)definite. In other words, a physically present dissipative effect such as velocity-dependent friction may destabilize the feedbacklinearized closed-loop system. The controller (25)-(33) proposed in this work ensures that such a case cannot happen at all because keeping the natural inertia $\left(\boldsymbol{\Lambda}_{i, \mathrm{des}}=\boldsymbol{\Lambda}_{i}\right)$ directly implies $\boldsymbol{\Lambda}_{i \text {,des }} \boldsymbol{\Lambda}_{i}^{-1} \boldsymbol{D}_{i}^{\text {fric }} \dot{\boldsymbol{x}}_{i}=\boldsymbol{D}_{i}^{\text {fric }} \dot{\boldsymbol{x}}_{i}$, that is, the dissipative nature of the friction is preserved.

Feedback linearization enforces a desired, constant inertial behavior. Frequently one chooses $\boldsymbol{\Lambda}_{i, \mathrm{des}}=\boldsymbol{I}$. As a consequence, the robot must react to external forces and torques in a way such that this inertial response is independent of the joint configuration. However, the natural dynamics of the system demand the opposite: the task-space inertias to be accelerated strongly depend on the joint configuration in practice. In Fig. 7 two different scenarios are depicted which yield largely different reflected inertias at the end-effector in vertical direction. If (60) represents the equations of motion in this Cartesian direction, for example, then the inertial reaction for feedback linearization has to be identical in both scenarios. If $\boldsymbol{\Lambda}_{i, \text { des }}=\boldsymbol{I}$ and $M_{\text {large }} \gg M_{\text {small }}$, then the configuration on the left of Fig. 7 leads to a large joint torque in joint 1 when interacting with the end-effector in vertical direction. That can cause problems in terms of actuator limitations when the maximum feasible torque is reached. This problem would not arise on the right side of Fig. 7. In other words, the accessible workspace of the robot for feedback linearization depends on the controller parametrization through $\boldsymbol{\Lambda}_{i, \mathrm{des}}$. Thus, the linear dynamics (60) may contradict the capabilities of the real robot. Moreover, the implementation of constant task-space inertias such as $\boldsymbol{\Lambda}_{i, \text { des }}=\boldsymbol{I}$ can be unintuitive. One would probably expect a lower acceleration if the apparent inertia is high. In the approach proposed here, the natural inertia is preserved $\left(\boldsymbol{\Lambda}_{i, \mathrm{des}}=\boldsymbol{\Lambda}_{i}\right)$ so that the problems described above cannot
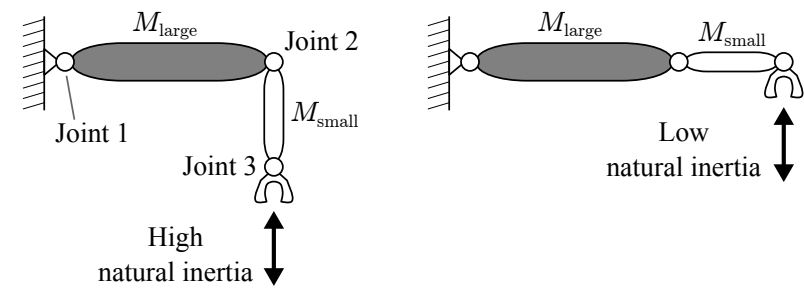

Fig. 7. Two different configurations for the joints 1,2 , and 3 , with largely different Cartesian inertias. On the left, the natural Cartesian inertia in vertical direction is high since the mass/inertia $M_{\text {large }}$ of the first link (shaded) has to be accelerated to move the end-effector. On the right, the end-effector can be moved also by accelerating the mass/inertia $M_{\text {small }}$ of the second link.

occur at all.

2) Disadvantages compared to feedback linearization: Feedback linearization yields exponential stability on all hierarchy levels. In the work proposed here, the proof only covers asymptotic stability of the overall equilibrium. However, it has to be noted that the exponential stability properties of (60) rely on the accurate knowledge of all dynamic parameters to be cancelled during feedback linearization. While compensation terms are also exploited in the controller presented here, the extent is significantly reduced compared to feedback linearization. During the latter, the Coriolis and centrifugal terms, for example, have to be completely annihilated, while that is not the case for (26) which is even a power-conserving feedback, also in the presence of modeling uncertainties. Another example is the feedback of the external forces and torques, as described above. In order to realize (60), $\boldsymbol{F}_{\dot{\boldsymbol{x}}_{i}}^{\text {ext }}$ has to be known accurately. The simulations in Section VI will demonstrate that a feedback-linearized system is particularly prone to such inaccuracies. Furthermore, a potential conflict of objectives exists when aiming at a specific transient behavior for feedback linearization. An exemplary case is the implementation of a desired damping ratio. While the task-space inertias can be chosen arbitrarily, the stiffness and damping gains also have a significant influence. If these are already defined by the subtasks themselves (e.g., through requirements on the desired contact stiffness and damping), then the advantages of exponential stability may not fully come into effect.

As shown in (60), the effect of cross-couplings related to external forces and torques is removed by the controller for feedback linearization. Depending on the choice (33) in the approach presented here, cross-couplings are either present (case 1), or they are also compensated for (case 2). Yet, if taskspace acceleration errors on higher-priority levels are non-zero in case 2, external disturbances can still influence the lowerpriority levels through the top-down disturbances related to (29). But if constant external task-space forces and torques are exerted, no such cross-coupling are encountered, similar to the case of feedback linearization. Moreover, in the simulations (Section VI) and the experiments (Section VII) it will be shown that the cross-couplings in the presented approach (case 1 and case 2) are small in practice, even during highly dynamic motions. Note again that the theoretically beneficial, complete external-force-torque-decoupling for feedback linearization relies on the accurate knowledge of $\boldsymbol{F}_{\dot{\boldsymbol{x}}_{i}}^{\text {ext }}$ or $\boldsymbol{\tau}^{\text {ext }}$, respectively. 


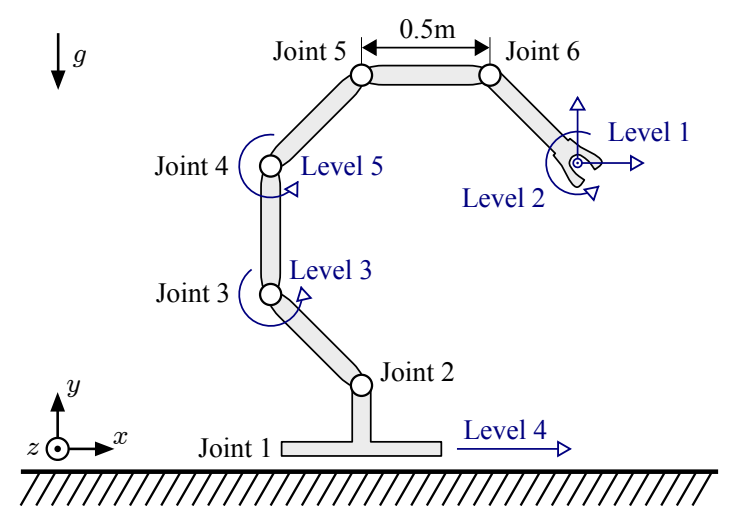

Fig. 8. Initial configuration of the planar simulation model with six DOF (joint 1: prismatic joint, joints 2-6: revolute joints). Gravity is simulated with $g=9.81 \frac{\mathrm{m}}{\mathrm{s}^{2}}$. Each link is modeled as a massless bar with a centered point mass of $1 \mathrm{~kg}$. The first link has a length of $0.25 \mathrm{~m}$, all other links have a length of $0.5 \mathrm{~m}$

It is well known that the identification and control feedback of the external disturbances can compromise the robustness of the closed loop in practice and is not trivial.

The pros and cons from Section V-B1 and Section V-B2 are condensed in Table I for a direct comparison.

\section{Simulations}

The controller is evaluated on a six-DOF planar manipulator in simulation as illustrated in Fig. 8. A task hierarchy with five priority levels is implemented with the task definitions and controller gains following Table II. On hierarchy level one, the full translational motion of the tool center point (TCP) in the plane is addressed. The second level is represented by the orientation of the TCP about the $z$-axis. Hierarchy level three covers the motion of the third joint, the translational motion of the mobile base in $x$-direction (first joint) is addressed on level four, and the lowest-priority level five specifies the motion of the fourth joint.

In order to compare the proposed controller (25)-(33) with the state of the art, a feedback linearization is simulated with the same desired stiffness and damping values (Table II). Furthermore, the desired task-space inertias for feedback linearization are chosen as $\boldsymbol{\Lambda}_{i \text {,des }}=\boldsymbol{I}$ for $i=1 \ldots 5$ in all of the simulations, following the common specification.

In simulation \#1 desired trajectories are applied on all hierarchy levels and the transient responses of the proposed controller and feedback linearization are analyzed in case of initial task-space errors. The desired reference trajectories are plotted in Fig. 9. The transients for both closed loops are depicted in Fig. 10. One can observe the exponential stability properties related to feedback linearization.

In simulation \#2 the control performance in the presence of various modeling errors and uncertainties is addressed. The simulated errors and uncertainties are defined as follows:

- The diagonal of the inertia matrix $\boldsymbol{M}(\boldsymbol{q})$ used in the control law is disturbed. On each element $7 \%$ of the original value in the initial configuration (see Fig. 8) is either added or subtracted, following the order --++-+ .
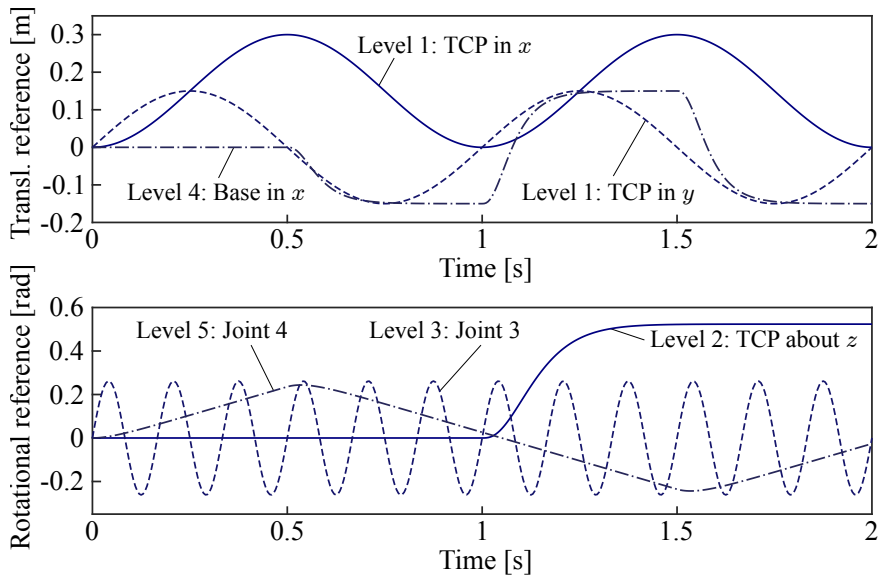

Fig. 9. Simulation \#1 (convergence): The top diagram depicts all translational reference trajectories to be tracked (levels 1,4 ), and the bottom diagram shows the corresponding rotational reference trajectories (levels 2, 3, 5). The zero point of the reference trajectories is defined by the desired initial configuration which is illustrated in Fig. 8 and described by the joint configuration $\left(0 \mathrm{~m}, 45^{\circ},-45^{\circ},-45^{\circ},-45^{\circ},-45^{\circ}\right)$.

- The Coriolis and centrifugal matrix $\boldsymbol{C}(\boldsymbol{q}, \dot{\boldsymbol{q}})$ used in the control law is reduced by $30 \%$ of the current value in each time step.

- Additional unmodeled joint friction is present in the simulated dynamics. All joints undergo viscous joint friction with the coefficients $0.05 \mathrm{Nms} / \mathrm{rad}$ and $0.05 \mathrm{Ns} / \mathrm{m}$, respectively.

The desired task-space stiffness and damping values on each hierarchy level are provided in Table II. The simulation results including the respective reference trajectories are shown in Fig. 11. One can see that the proposed controller is able to track the trajectories well. Although feedback linearization uses the same controller gains for stiffness and damping, large errors can be observed, particularly on the levels 2 and 5. A more detailed comparison of the task-space tracking errors of the simulation results is provided in Fig. 12. It is known from the literature that especially modeling errors in the inertia matrix deteriorate the control performance of operationalspace controllers [17]. Both the proposed controller and a feedback linearization belong to this category. But a feedback linearization appears to be particularly prone to errors related to $\boldsymbol{M}(\boldsymbol{q})$. This might be due to the explicit cancellation of the natural inertia by model-based feedback control and the subsequent implementation of a constant, diagonal, and decoupled task-space inertia.

Since simulation \#2 represents only one specific controller parametrization and uncertainty scenario, 2000 additional simulations with random modeling errors and uncertainties have been conducted, with these disturbances lying in the following ranges:

- The diagonal of the inertia matrix $\boldsymbol{M}(\boldsymbol{q})$ used in the control law is disturbed. Between $0 \%$ and $7 \%$ of the original value in the initial configuration is individually and randomly added to each element.

- The Coriolis and centrifugal matrix $\boldsymbol{C}(\boldsymbol{q}, \dot{\boldsymbol{q}})$ used in the control law is disturbed between $-10 \%$ and $10 \%$ of the 
TABLE I

DIRECT COMPARISON BETWEEN THE PROPOSED APPROACH (25)-(33) (CASES 1 AND 2) AND FEEDBACK LINEARIZATION

\begin{tabular}{|l||c|c|c|}
\hline Property & Feedback linearization & Control (25)-(33), case 1 & Control (25)-(33), case 2 \\
\hline \hline Stability & Exponential & Asymptotic & Asymptotic \\
\hline Control feedback of external forces/torques & Yes & No & Yes \\
\hline Cross-couplings (const. external forces/torques) & No & Yes & No \\
\hline Inertial behavior & Desired inertia (constant) & Natural inertia & Natural inertia \\
\hline Effect of unmodeled friction & Potential source of instability & Dissipative & Dissipative \\
\hline Cancellation of Cor./centr. terms & Not power-conserving & Power-conserving & Power-conserving \\
\hline
\end{tabular}

TABLE II

TASK DESCRIPTIONS AND CONTROLLER GAINS FOR THE SIMULATION

\begin{tabular}{llcc}
\hline Lvl. & $\begin{array}{l}\text { Task } \\
\text { description }\end{array}$ & $\begin{array}{c}\text { Sim.\#1 } \\
\text { stiffness/damping }\end{array}$ & $\begin{array}{c}\text { Sim. \#2 and \#3 } \\
\text { stiffness/damping }\end{array}$ \\
\hline 1 & TCP in $x$ & $200 \frac{\mathrm{N}}{\mathrm{m}} / 10 \frac{\mathrm{Ns}}{\mathrm{m}}$ & $500 \frac{\mathrm{N}}{\mathrm{m}} / 50 \frac{\mathrm{Ns}}{\mathrm{m}}$ \\
1 & TCP in $y$ & $200 \frac{\mathrm{N}}{\mathrm{m}} / 10 \frac{\mathrm{Ns}}{\mathrm{m}}$ & $500 \frac{\mathrm{N}}{\mathrm{m}} / 50 \frac{\mathrm{Ns}}{\mathrm{m}}$ \\
2 & TCP about $z$ & $50 \frac{\mathrm{Nm}}{\mathrm{rd}} / 5 \frac{\mathrm{Nms}}{\mathrm{rad}}$ & $200 \frac{\mathrm{Nm}}{\mathrm{rad}} / 40 \frac{\mathrm{Nms}}{\mathrm{rad}}$ \\
3 & Joint 3 about $z$ & $50 \frac{\mathrm{Nm}}{\mathrm{rad}} / 5 \frac{\mathrm{Nm}}{\mathrm{rd}}$ & $200 \frac{\mathrm{Nm}}{\mathrm{rad}} / 40 \frac{\mathrm{Nms}}{\mathrm{rd}}$ \\
4 & Joint 1 in $x$ & $100 \frac{\mathrm{N}}{\mathrm{m}} / 10 \frac{\mathrm{Ns}}{\mathrm{m}}$ & $400 \frac{\mathrm{N}}{\mathrm{m}} / 250 \frac{\mathrm{Ns}}{\mathrm{m}}$ \\
5 & Joint 4 about $z$ & $50 \frac{\mathrm{Nm}}{\mathrm{rad}} / 5 \frac{\mathrm{Nms}}{\mathrm{rad}}$ & $200 \frac{\mathrm{Nm}}{\mathrm{rad}} / 40 \frac{\mathrm{Nms}}{\mathrm{rad}}$ \\
\hline
\end{tabular}

current value in each time step.

- Additional unmodeled joint friction is present in the simulated dynamics. All joints undergo viscous joint friction with the coefficients between 0 and $0.02 \mathrm{Nms} / \mathrm{rad}$ and $\mathrm{Ns} / \mathrm{m}$, respectively.

Figure 13 shows the average and maximum task-space errors for all 2000 simulation runs of $6 \mathrm{~s}$ each.

In simulation \#3 various external disturbances are applied and the transient responses are analyzed. The top diagram in Fig. 14 shows these interactions. Between $0.5 \mathrm{~s}$ and $1.5 \mathrm{~s}$ external forces are exerted on the end-effector in the $x$ - and $y$-direction, with $30 \mathrm{~N}$ and $40 \mathrm{~N}$, respectively. Between $3 \mathrm{~s}$ and $5 \mathrm{~s}$, a force of $50 \mathrm{~N}$ is applied to the mobile base in $x$-direction, and between $7.5 \mathrm{~s}$ and $8.5 \mathrm{~s}$, a torque of $30 \mathrm{Nm}$ excites the third joint. The diagrams 2-7 illustrate the influence on the task-space errors on all hierarchy levels. The shaded areas describe the intervals on the respective priority levels of the disturbance. Both the proposed controller and feedback linearization are simulated with and without control feedback of external forces and torques. One can observe that the proposed controller without feedback of these disturbances features a similar behavior as its counterpart since the crosscouplings onto the other hierarchy levels are comparatively small. It is noticeable that the feedback-linearized system without the active feedback of the external forces and torques shows an inferior tracking performance during all external interactions. Beside the task-space errors one can also analyze the level-specific energies to assess the control performance. Figure 15 depicts the energies consisting of the respective virtual potentials and kinetic energies related to each priority level. Again, the shaded areas illustrate the time intervals on the particular levels where the external forces/torques are applied. By means of the low peak virtual energies on the other levels one can observe that external forces exerted on
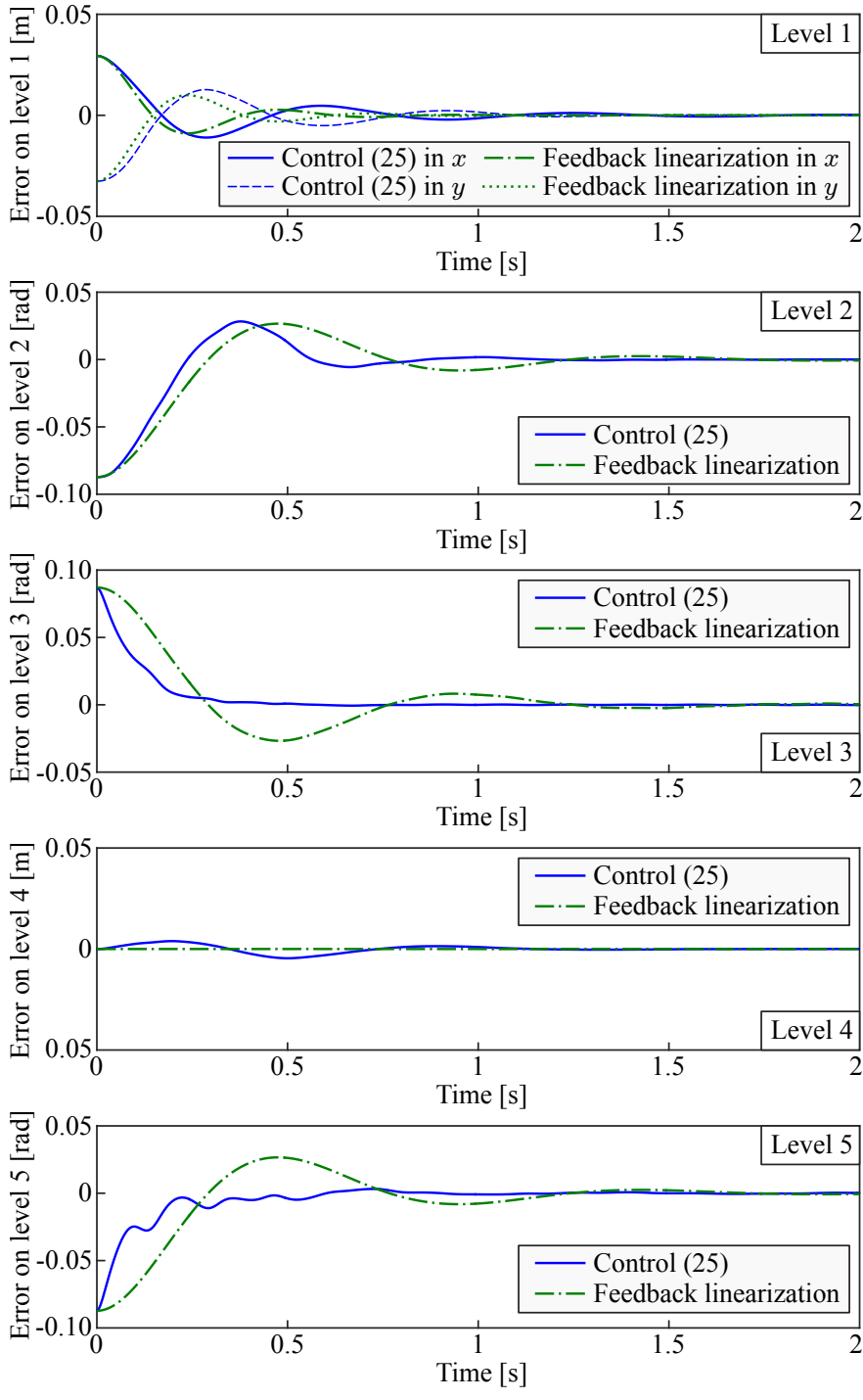

Fig. 10. Simulation \#1 (convergence): Direct comparison between the proposed controller and feedback linearization for initial task-space errors and active desired trajectories in the absence of external forces and torques. The initial configuration of the robot at rest is described by the joint positions $\left(0 \mathrm{~m}, 40^{\circ},-40^{\circ},-50^{\circ},-40^{\circ},-50^{\circ}\right)$.

one hierarchy level do not significantly disturb the other levels despite the fast and dynamic motions. The only exception is the feedback-linearized system without active feedback of the external forces and torques. Due to the large values, the virtual energies related to this controller are not contained in Fig. 15 

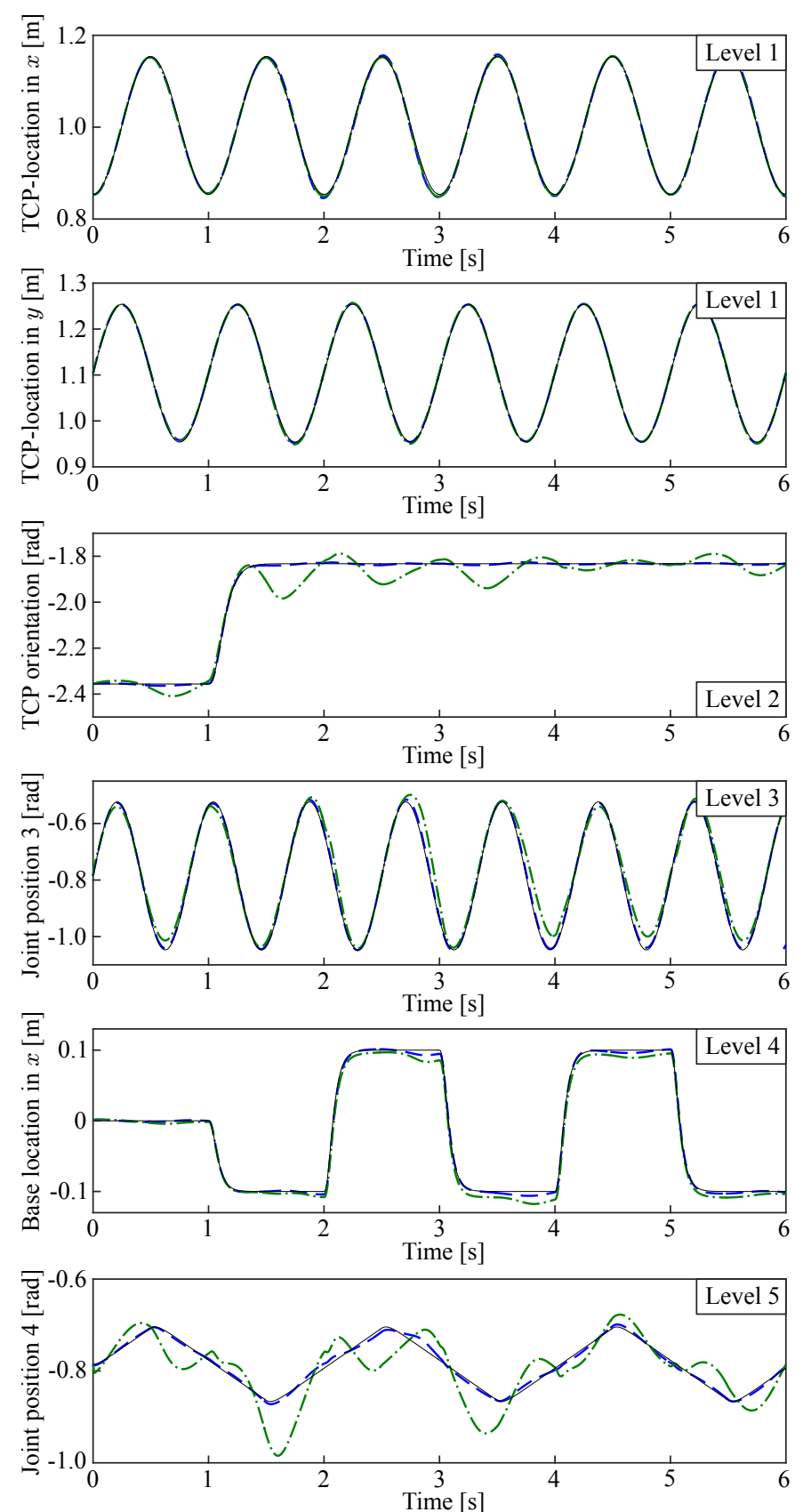

- Reference $\quad-\quad$ Control (25) - - - Feedback linearization

Fig. 11. Simulation \#2 (modeling uncertainties): Trajectory tracking performance of the proposed controller and feedback linearization in the presence of modeling errors and uncertainties

but outsourced to Fig. 16. Note that there are differences of several orders of magnitude between classical feedback linearization without use of the external forces/torques and the corresponding proposed controller (case 1, see (33)).

In this context it has to be noted that feedback linearization with control feedback of the external forces/torques is rather sensitive to inaccuracies in these terms. If one introduces an error of only $5 \%$ (for example by feedback of $95 \%$ of the external forces/torques for feedback linearization of simulation \#3), then the control performance already degrades
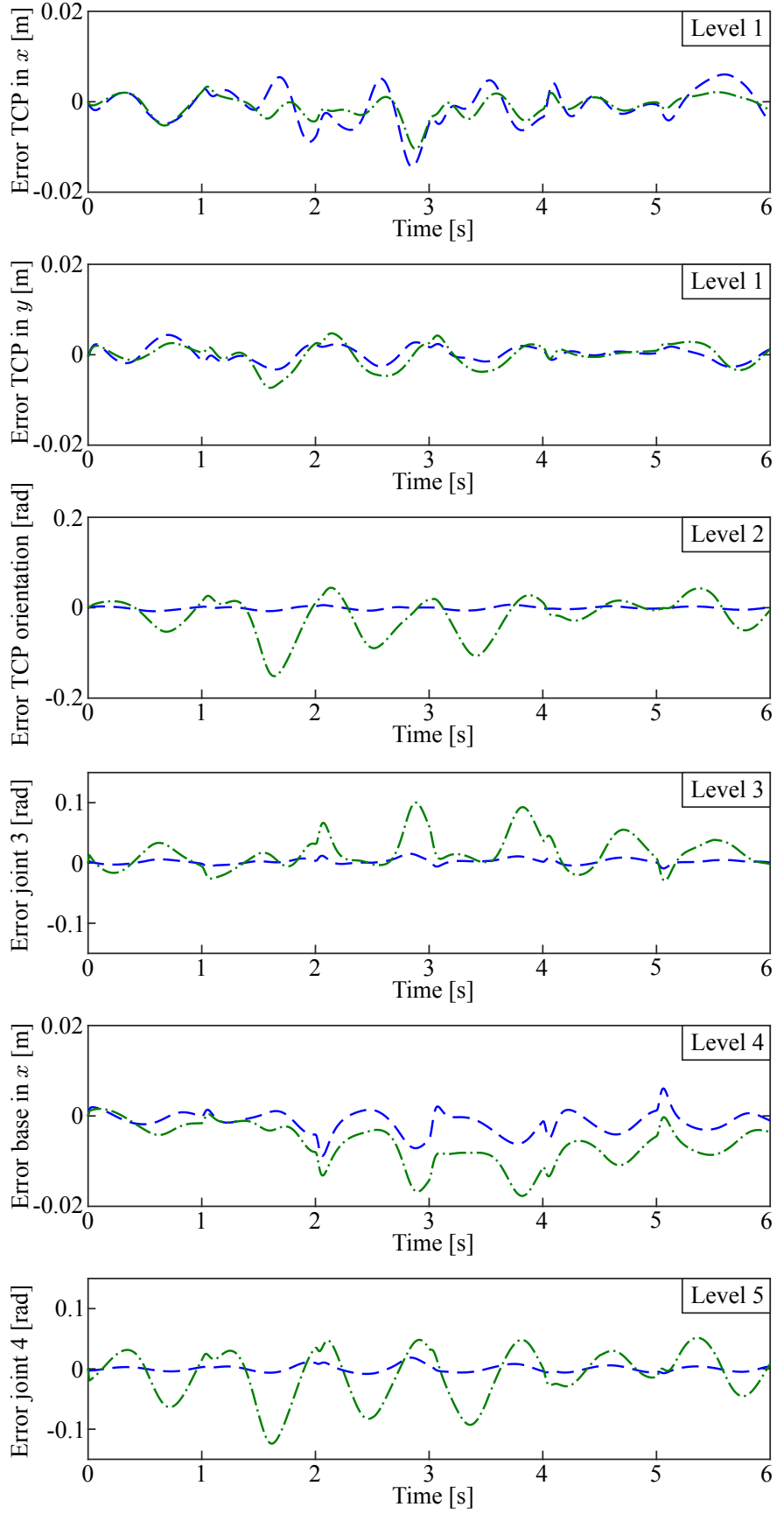

-- Control (25) - - - Feedback linearization

Fig. 12. Simulation \#2 (modeling uncertainties): Task-space errors on all hierarchy levels in the presence of modeling errors and uncertainties

significantly such that the peak virtual energy goes up to about $13 \mathrm{~J}$ instead of less than $3 \mathrm{~J}$ as before. In practice the feedback of external forces and torques is difficult. Beside the general question of availability, the accuracy of the measurements or estimations is known to be problematic. Therefore, the high control performance of the proposed approach (case 1 in (33), without feedback of external forces/torques) particularly gains relevance for practical applications. 

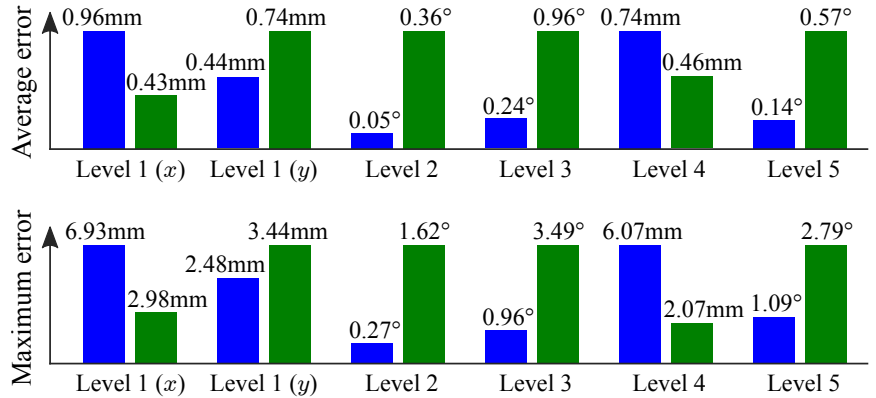

Control (25) Feedback linearization

Fig. 13. Simulation \#2 (modeling uncertainties): Average and maximum taskspace errors of 2000 simulations with random disturbances

TABLE III

TASKS AND CONTROL GAINS IN THE EXPERIMENTS \#1 AND \#2. $(\dagger$ : DAMPING RATIO)

\begin{tabular}{clcc}
\hline Level & Task description & Stiffness & Damping \\
\hline 1 & Transl. Cart. impedance (TCP) & $1500 \mathrm{~N} / \mathrm{m}$ & $0.7^{\dagger}$ \\
2 & Rot. Cart. impedance (TCP) & $200 \mathrm{Nm} / \mathrm{rad}$ & $0.7^{\dagger}$ \\
3 & Joint impedance (joint 1) & $300 \mathrm{Nm} / \mathrm{rad}$ & $30 \mathrm{Nms} / \mathrm{rad}$ \\
\hline
\end{tabular}

\section{EXPERIMENTS}

The controller is evaluated in two experiments on a commercially available KUKA LWR IV+, a torque-controlled lightweight robot with seven DOF, see Fig. 17. The translational Cartesian impedance at the TCP with $m_{1}=3$ is chosen as task on the first-priority level, while the rotational Cartesian impedance at the TCP with $m_{2}=3$ characterizes hierarchy level two. ${ }^{5}$ The lowest-priority level is defined by a joint impedance realized in the first joint with $m_{3}=1$. This three-level task hierarchy and the control gains for the two experiments are summarized in Table III. The X- and Yaxes illustrated in Fig. 17 describe the motion of the TCP in the horizontal plane, respectively, and the Z-axis defines the vertical motion.

In experiment \#1 a synchronized translational reference trajectory in all three TCP-directions is applied (level 1), whereas the orientation of the TCP is assigned to be maintained as best as possible (level 2). Moreover, the first joint is commanded to move in a range of about $23^{\circ}$ (level 3). The desired trajectories and the tracking performance can be observed in Fig. 18 . The zero positions and orientations in the diagrams represent the initial, non-singular configuration depicted in Fig. 17.6 Although the deviations between the desired trajectories and the actual task-space coordinates are minor, the errors are non-zero. That effect is expected in the presence of modeling uncertainties as in all impedance-based controllers.

${ }^{5}$ Note that the implemented TCP orientation control is based on a singularity-free quaternion representation. In the analysis of the results, Euler angles are utilized for the sake of intuitive interpretation. Damping ratios are implemented on the first two levels instead of a constant damping.

${ }^{6}$ Using the standard definitions of the joint angles on the KUKA LWR IV+, the initial configuration is described by $(-0.7,-0.554,0.22,1.45,-0.24,-1.1,0.4) \mathrm{rad}$.
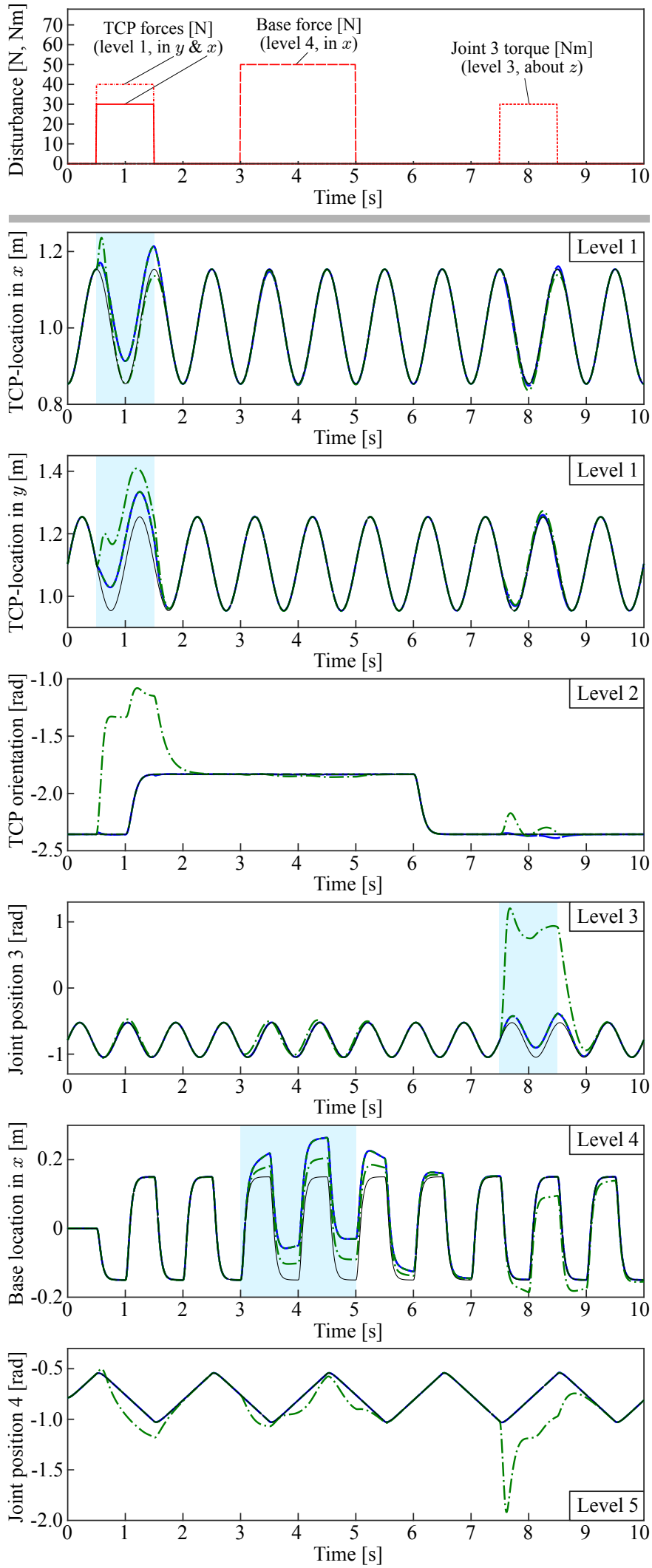

- Reference trajectory

- Control (25), case 1, without feedback of external forces/torques

--- Control (25), case 2, with feedback of external forces/torques

-.- Feedback linearization, without feedback of external forces/torques

-.-- Feedback linearization, with feedback of external forces/torques

Fig. 14. Simulation \#3 (physical interaction): Task-space errors occurring during physical interaction with the robot 

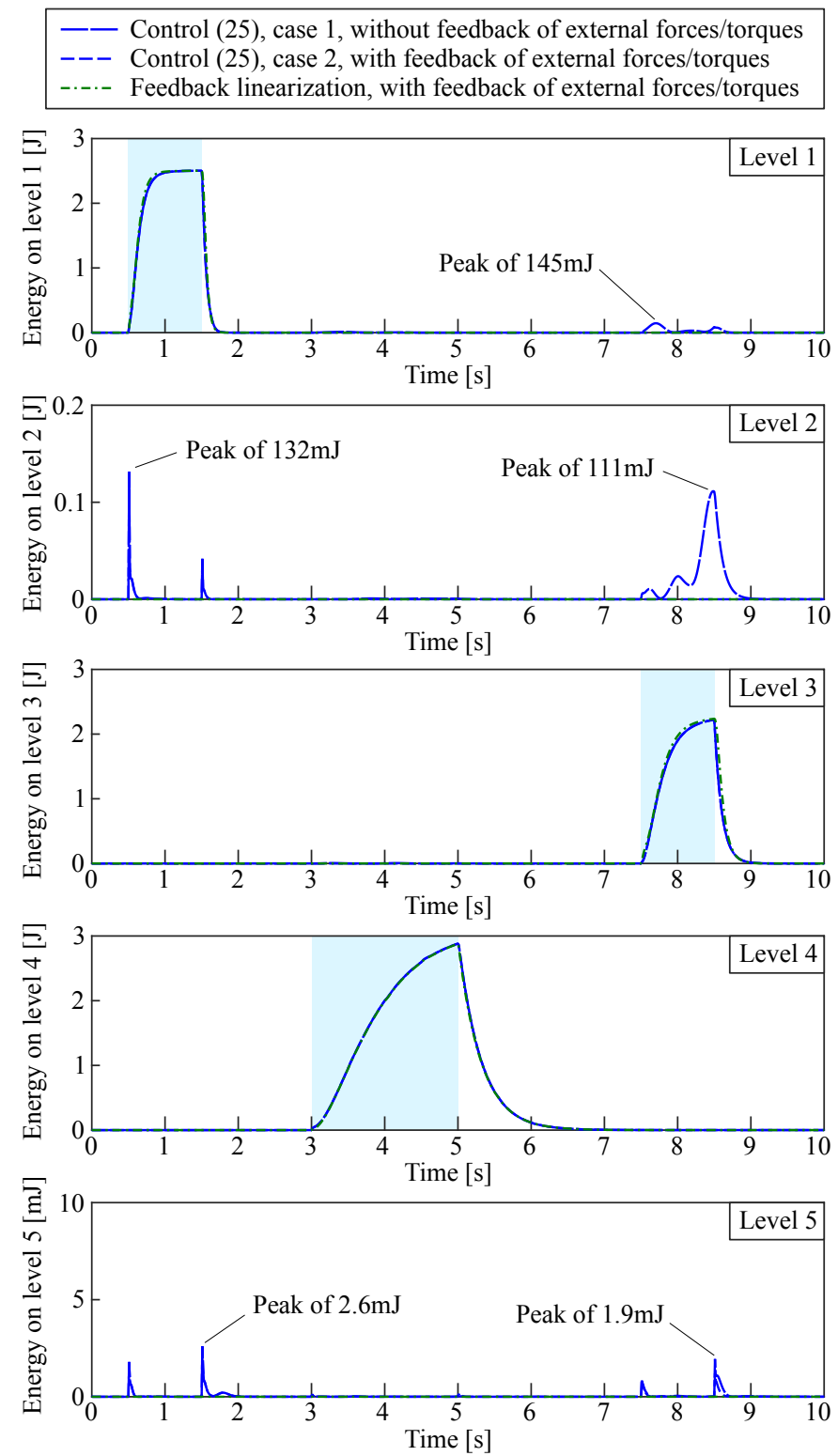

Fig. 15. Simulation \#3 (physical interaction): Level-specific energies during physical interaction with the robot

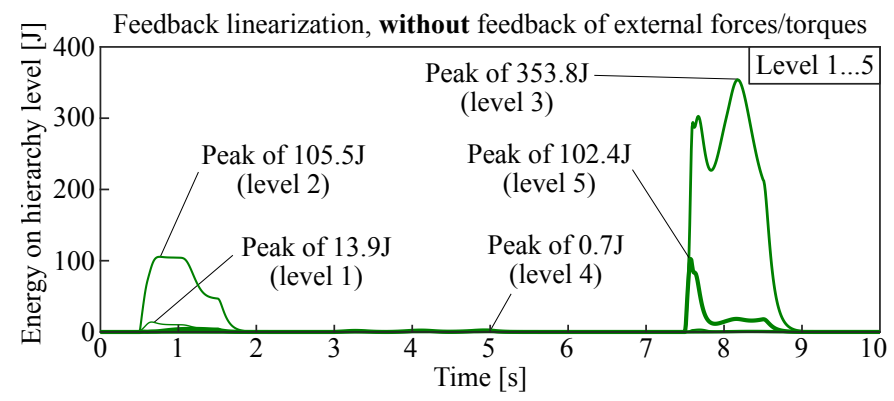

Fig. 16. Simulation \#3 (physical interaction): Level-specific energies during physical interaction with the robot for feedback linearization. No feedback of external forces and torques is used in the controller.

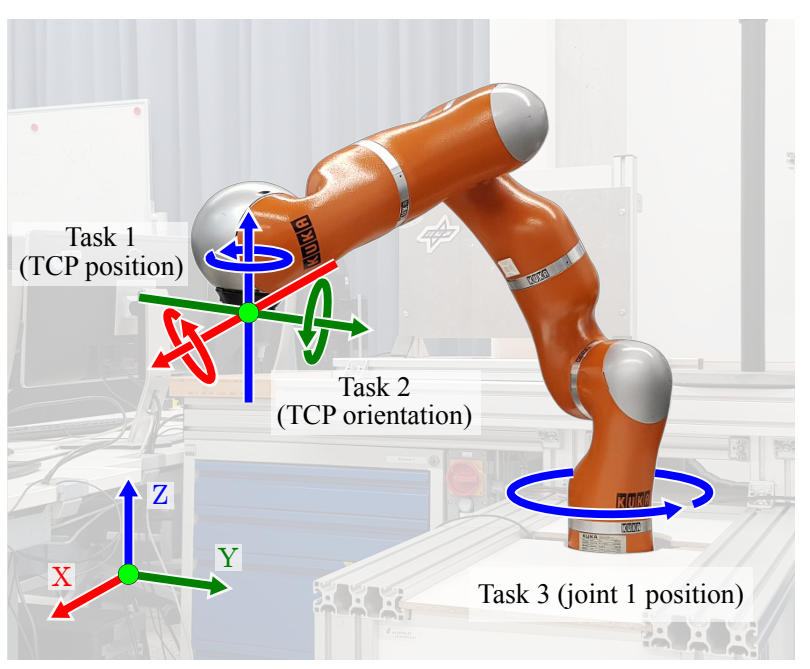

Fig. 17. Setup for the experimental evaluation: KUKA LWR IV+ with threelevel task hierarchy
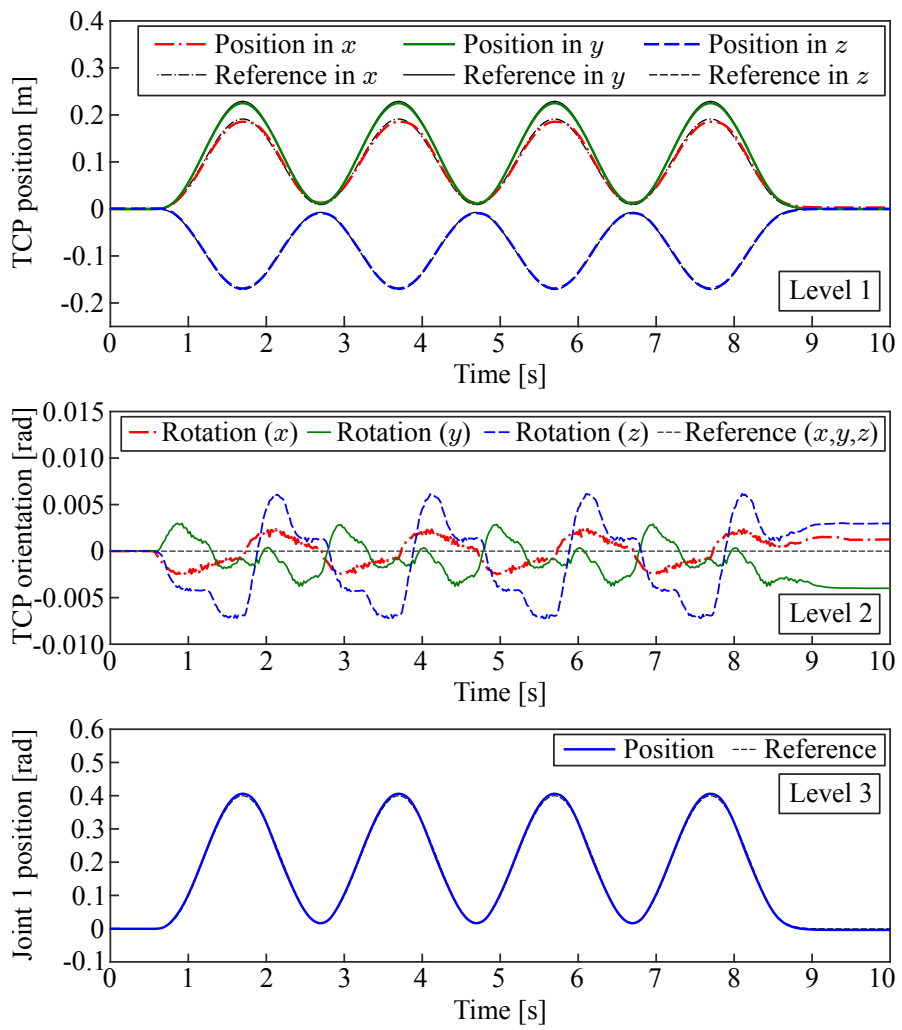

Fig. 18. Experiment \#1 (trajectory tracking): The values plotted in the second diagram describe the Euler angles obtained from successively rotating about the $\mathrm{X}-, \mathrm{Y}-$, and $\mathrm{Z}$-axis.

To assess the control performance more accurately, Fig. 19 shows the task-space errors from Fig. 18, where the leveltwo error is depicted as the total orientation error, that is, the absolute angle between the actual and the desired orientation of the TCP. At the end of the trajectory $(t>10 \mathrm{~s})$, a small steady-state error remains as discussed above. Increasing the desired stiffness values in Table III would reduce this error at the cost of less compliance in case of physical interaction. Additionally, the controller is implemented without the $\gamma$ - 

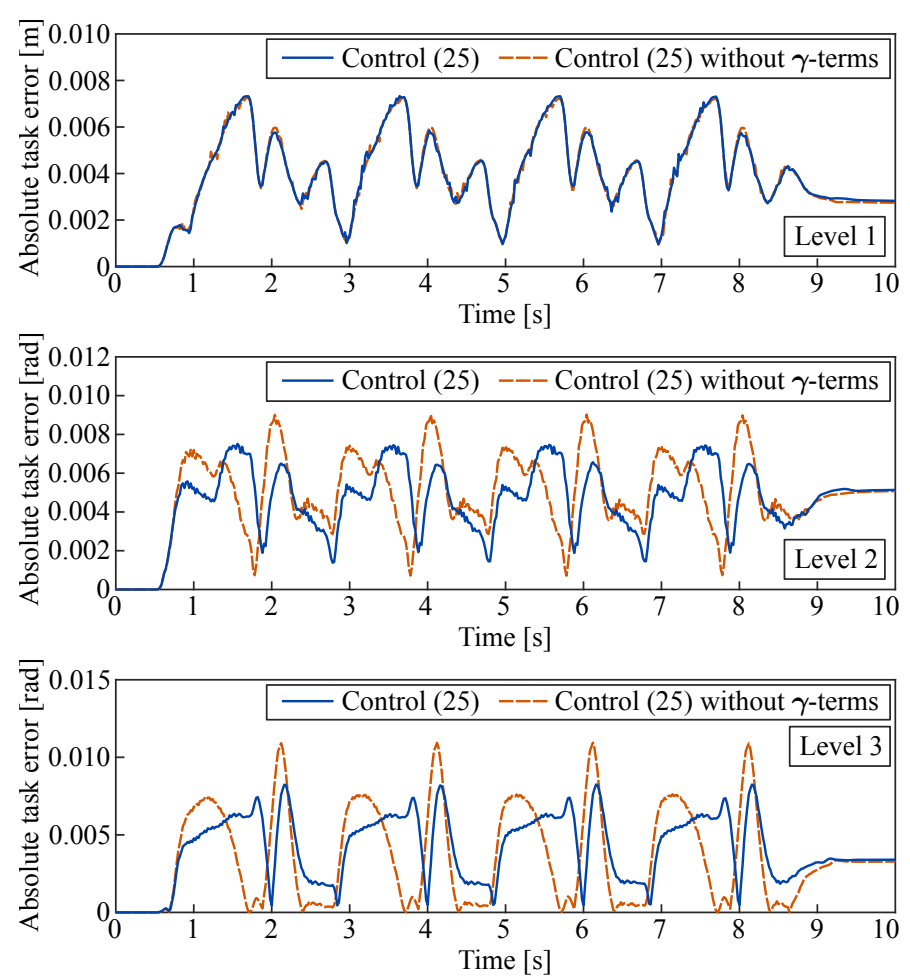

Fig. 19. Experiment \#1 (trajectory tracking): Task-space errors on the three hierarchy levels. The results for the control law without $\gamma$-terms in (32) are plotted additionally to observe the effect of the top-down disturbance.

components in (32) so that the effect of the top-down disturbance can be observed in practice. As the theory has shown, no effect on the first priority level is expected, which is confirmed by the results in Fig. 19 (top diagram). On the lower-priority levels, the $\gamma$-terms in (32) reduce the maximum task-space errors by about $16 \%$ (level 2, center diagram) and $25 \%$ (level 3 , bottom diagram).

Note that feedback-linearization-based approaches are prone to the same effects of steady-state errors and non-zero taskspace errors during motion because they also constitute PD control laws basically. That is an inevitable consequence of the realization of a desired contact stiffness.

In experiment \#2 different types of external disturbances are exerted on the robot through physical human-robot interaction while the desired trajectories from experiment \#1 are applied with smaller velocities. Note that the controller for case 1 in (33) is considered, that is, no external forces/torques are used in the control feedback. The external torque $\tau^{\text {ext }}$ consulted in the analysis of the results is estimated using a momentumbased observer [41] and subsequently mapped to the external task-space forces and torques acting on the three hierarchy levels. The total force on level one, i. e., the Euclidean norm of the three Cartesian external forces at the TCP, is depicted in diagram 1 of Fig. 20. The total external torque about the TCP is shown in diagram 4 , and the external torque exerted on the first joint can be observed in diagram 7 .

After about $2.5 \mathrm{~s}$ a force of about $89 \mathrm{~N}$ is applied at the TCP and superposed by a TCP torque of about $5 \mathrm{Nm}$. These effects are highlighted by shaded rectangles. As desired, that leads to a deviation on the first two hierarchy levels. In the energy plot on level three (bottom diagram) one can see that no relevant cross-coupling is present as the peak value is only about $14 \mathrm{~mJ}$. The second disturbance after about $11.5 \mathrm{~s}$ acts only on the first joint with a torque of about $27 \mathrm{Nm}$. The energy plots for the levels one and two reveal that this external torque on level three does not affect the higher-priority tasks in a noticeable way ( $80 \mathrm{~mJ}$ on level one, $4 \mathrm{~mJ}$ on level two). After about $22 \mathrm{~s}$, a third external disturbance is applied which acts on all hierarchy levels yielding peaks in all level energies. The experiment illustrates that physical compliance is achieved by the impedance control law and external force-torque crosscouplings are small in practice.

\section{CONCLUSIONS}

The classical approach to perform multiple robotic manipulation tasks simultaneously is to dynamically decouple all of them and feedback-linearize the system. Both the common inverse-dynamics approaches and the Operational Space Formulation (OSF) follow this procedure, finally leading to equivalent results. While desired task-space trajectories can be ideally tracked in theory that way, the controller relies on accurate model knowledge in practice. Moreover, in order to implement a specified contact stiffness and damping, one has to measure or estimate the external forces/torques and use them in the control feedback. Here, we provided a hierarchical multi-objective tracking controller for an arbitrary number of priority levels which features asymptotic stability of the equilibrium and it gets along without any feedback of external forces and torques. Furthermore, a dedicated contact impedance can be specified and realized, and the natural inertia of the robot is preserved. Simulations and experiments with multiple hierarchy levels have been conducted to evaluate the approach and validate the theoretical findings obtained from the proof of asymptotic stability. The presented controller is predestined for precise task-space trajectory tracking while it implements a desired contact impedance for physical interaction at the same time.

The next steps will cover extensive experimental evaluations of the controller in terms of trajectory tracking and interaction scenarios, and the analysis of the robustness against modeling uncertainties and disturbances. Moreover, the case of an inconsistent task hierarchy will be addressed, e.g., when the overall task dimension exceeds the number of available DOF in the system.

\section{APPENDIX A}

In the following the descriptions for stability, uniform stability, uniform attraction, and uniform asymptotic stability are recapitulated in Definition 1, while their restricted versions conditionally to subsets are specified in Definition 2.

Definition 1: [26], [34], [42] The equilibrium point $\boldsymbol{z}=\mathbf{0}$ of a system (47) is

- stable if for each $\xi>0$, there is $\delta=\delta\left(\xi, t_{0}\right)>0$ such that

$$
\left\|\boldsymbol{z}_{0}\right\|<\delta \Rightarrow\|\boldsymbol{z}(t)\|<\xi, \quad \forall t \geq t_{0} \geq 0 ;
$$


- uniformly stable (US) if for each $\xi>0$, there is $\delta=\delta(\xi)>0$, independent of $t_{0}$, such that (61) is satisfied;

- uniformly attractive (UA) if there is a positive constant $c$, independent of $t_{0}$, such that $\boldsymbol{z}(t) \rightarrow \mathbf{0}$ as $t \rightarrow \infty$ uniformly in $t_{0}$, for all $\left\|\boldsymbol{z}_{0}\right\|<c$. That is, for each $\eta>0$, there is $T=T(\eta)>0$ such that

$$
\|\boldsymbol{z}(t)\| \leq \eta, \forall t \geq t_{0}+T, \forall t_{0} \geq 0, \forall\left\|\boldsymbol{z}_{0}\right\|<c ;
$$

- uniformly asymptotically stable (UAS) if it is US and UA.

Definition 2: [26] Let $\mathcal{Z} \subset \mathbb{R}^{n}$ contain $\boldsymbol{z}=\mathbf{0}$. The point $\boldsymbol{z}=\mathbf{0}$ of the system (47) is

- uniformly stable conditionally to $\mathcal{Z}$ (Z्Z-US) if, for each $\xi>0$, there exists $\delta=\delta(\xi)>0$, independent of $t_{0}$, such that

$$
\forall \boldsymbol{z}_{0} \in \mathcal{Z} \text { and }\left\|\boldsymbol{z}_{0}\right\|<\delta \Rightarrow\|\boldsymbol{z}(t)\|<\xi
$$

for all $t \geq t_{0} \geq 0$.

- uniformly attractive conditionally to $\mathcal{Z}$ (Z-UA) if, for each $\eta>0$, there is $T=T(\eta)>0$ such that

$$
\|\boldsymbol{z}(t)\| \leq \eta, \quad \forall t \geq t_{0}+T, \quad \forall t_{0} \geq 0
$$

for all $\left\|\boldsymbol{z}_{0}\right\|<c$ and $\boldsymbol{z}_{0} \in \mathcal{Z}$.

- uniformly asymptotically stable conditionally to $\mathcal{Z}(\mathcal{Z}$ UAS) if it is $\mathcal{Z}$-US and $\mathcal{Z}$-UA.

\section{ACKNOWLEDGEMENTS}

The authors would like to thank Xuwei Wu and Nico Mansfeld for their hardware support during the experiments and Martin Görner for the drawing in Fig. 1. This work was partially supported by the Bavarian Ministry of Economic Affairs, Regional Development and Energy, within the project SMiLE2gether (LABAY102).

\section{REFERENCES}

[1] Y. Nakamura, H. Hanafusa, and T. Yoshikawa, "Task-Priority Based Redundancy Control of Robot Manipulators," International Journal of Robotics Research, vol. 6, no. 2, pp. 3-15, June 1987.

[2] J. H. Hollerbach and K. C. Suh, "Redundancy Resolution of Manipulators through Torque Optimization," IEEE Journal of Robotics and Automation, vol. RA-3, no. 4, pp. 308-316, August 1987.

[3] B. Siciliano and J.-J. Slotine, "A General Framework for Managing Multiple Tasks in Highly Redundant Robotic Systems," in Proc. of the 5th International Conference on Advanced Robotics, June 1991, pp. $1211-1216$.

[4] O. Kanoun, F. Lamiraux, and P.-B. Wieber, "Kinematic Control of Redundant Manipulators: Generalizing the Task-Priority Framework to Inequality Task," IEEE Transactions on Robotics, vol. 27, no. 4, pp. 785-792, August 2011.

[5] A. Escande, N. Mansard, and P.-B. Wieber, "Hierarchical quadratic programming: Fast online humanoid-robot motion generation," International Journal of Robotics Research, vol. 33, no. 7, pp. 1006-1028, June 2014.

[6] A. Herzog, N. Rotella, S. Mason, F. Grimminger, S. Schaal, and L. Righetti, "Momentum control with hierarchical inverse dynamics on a torque-controlled humanoid," Autonomous Robots, vol. 40, no. 3, pp. 473-491, March 2016.

[7] D. J. Agravante, G. Claudio, F. Spindler, and F. Chaumette, "Visual Servoing in an Optimization Framework for the Whole-Body Control of Humanoid Robots," IEEE Robotics and Automation Letters, vol. 2, no. 2, pp. 608-615, April 2017.
[8] F. L. Moro, M. Gienger, A. Goswami, N. G. Tsagarakis, and D. G. Caldwell, "An Attractor-based Whole-Body Motion Control (WBMC) System for Humanoid Robots," in Proc. of the 13th IEEE-RAS International Conference on Humanoid Robots, October 2013, pp. 42-49.

[9] J. Salini, V. Padois, and P. Bidaud, "Synthesis of Complex Humanoid Whole-Body Behaviors: a Focus on Sequencing and Tasks Transitions," in Proc. of the 2011 IEEE International Conference on Robotics and Automation, May 2011, pp. 1283-1290.

[10] N. Dehio, R. F. Reinhart, and J. J. Steil, "Multiple Task Optimization with a Mixture of Controllers for Motion Generation," in Proc. of the 2015 IEEE/RSJ International Conference on Intelligent Robots and Systems, September/October 2015, pp. 6416-6421.

[11] K. Bouyarmane and A. Kheddar, "On Weight-Prioritized Multitask Control of Humanoid Robots," IEEE Transactions on Automatic Control, vol. 63, no. 6, pp. 1632-1647, June 2018.

[12] O. Khatib, "A Unified Approach for Motion and Force Control of Robot Manipulators: The Operational Space Formulation," IEEE Journal of Robotics and Automation, vol. RA-3, no. 1, pp. 43-53, February 1987.

[13] — , "Inertial Properties in Robotic Manipulation: An Object-Level Framework," International Journal of Robotics Research, vol. 14, no. 1, pp. 19-36, February 1995.

[14] L. Righetti, J. Buchli, M. Mistry, and S. Schaal, "Inverse Dynamics Control of Floating-Base Robots with External Constraints: a Unified View," in Proc. of the 2011 IEEE International Conference on Robotics and Automation, May 2011, pp. 1085-1090.

[15] L. Sentis and O. Khatib, "Synthesis of Whole-Body Behaviors through Hierarchical Control of Behavioral Primitives," International Journal of Humanoid Robotics, vol. 2, no. 4, pp. 505-518, January 2005.

[16] L. Sentis, J. Park, and O. Khatib, "Compliant Control of Multicontact and Center-of-Mass Behavior in Humanoid Robots," IEEE Transactions on Robotics, vol. 26, no. 3, pp. 483-501, June 2010.

[17] J. Nakanishi, R. Cory, M. Mistry, J. Peters, and S. Schaal, "Operational Space Control: A Theoretical and Empirical Comparison," International Journal of Robotics Research, vol. 27, no. 6, pp. 737-757, June 2008.

[18] G. Antonelli, "Stability Analysis for Prioritized Closed-Loop Inverse Kinematic Algorithms for Redundant Robotic Systems," IEEE Transactions on Robotics, vol. 25, no. 5, pp. 985-994, October 2009.

[19] A. Karami, H. Sadeghian, M. Keshmiri, and G. Oriolo, "Hierarchical tracking task control in redundant manipulators with compliance control in the null-space," Mechatronics, vol. 55, pp. 171-179, November 2018.

[20] A. D. Ames and M. Powell, "Towards the Unification of Locomotion and Manipulation through Control Lyapunov Functions and Quadratic Programs," in Control of Cyber-Physical Systems, March 2013, pp. 219240.

[21] H. Sadeghian, L. Villani, M. Keshmiri, and B. Siciliano, "Task-Space Control of Robot Manipulators With Null-Space Compliance," IEEE Transactions on Robotics, vol. 30, no. 2, pp. 493-506, April 2014.

[22] E. Zergeroglu, D. D. Dawson, I. W. Walker, and P. Setlur, "Nonlinear Tracking Control of Kinematically Redundant Robot Manipulators," IEEE/ASME Transactions on Mechatronics, vol. 9, no. 1, pp. 129-132, March 2004.

[23] A. Dietrich, C. Ott, and A. Albu-Schäffer, "Multi-Objective Compliance Control of Redundant Manipulators: Hierarchy, Control, and Stability," in Proc. of the 2013 IEEE/RSJ International Conference on Intelligent Robots and Systems, November 2013, pp. 3043-3050.

[24] C. Ott, A. Dietrich, and A. Albu-Schäffer, "Prioritized Multi-Task Compliance Control of Redundant Manipulators," Automatica, vol. 53, pp. 416-423, March 2015.

[25] N. Hogan, "Impedance Control: An Approach to Manipulation: Part I - Theory, Part II - Implementation, Part III - Applications," Journal of Dynamic Systems, Measurement, and Control, vol. 107, pp. 1-24, March 1985.

[26] Z. M. Wang, Y. Tan, G. X. Wang, and D. Nešić, "On Stability Properties of Nonlinear Time-Varying Systems by Semi-definite Time-Varying Lyapunov Candidates," in Proc. of the 17th World Congress of the International Federation of Automatic Control, July 2008, pp. 11231128.

[27] A. Teel, E. Panteley, and A. Loría, "Integral Characterizations of Uniform Asymptotic and Exponential Stability with Applications," Mathematics of Control, Signals, and Systems, vol. 15, no. 3, pp. 177-201, July 2002.

[28] R. M. Murray, Z. Li, and S. S. Sastry, A Mathematical Introduction to Robotic Manipulation. CRC Press, 1994.

[29] A. Dietrich, C. Ott, and A. Albu-Schäffer, "An overview of null space projections for redundant, torque-controlled robots," International Journal of Robotics Research, vol. 34, no. 11, pp. 1385-1400, Sept. 2015. 
[30] S. Stramigioli, "Energy-Aware Robotics," in Mathematical Control Theory I, ser. Lecture Notes in Control and Information Sciences 461, M. K. Camlibel, A. A. Julius, R. Pasumarthy, and J. M. A. Scherpen, Eds. Springer International Publishing Switzerland, 2015, ch. 3, pp. 37-50.

[31] L. L. Whitcomb, A. A. Rizzi, and D. E. Koditschek, "Comparative Experiments with a New Adaptive Controller for Robot Arms," IEEE Transactions on Robotics and Automation, vol. 9, no. 1, pp. 59-70, February 1993.

[32] S. Boyd and L. Vandenberghe, Convex Optimization. Cambridge University Press, 2004.

[33] A. Iggidr, B. Kalitine, and R. Outbib, "Semidefinite Lyapunov Functions Stability and Stabilization," Mathematics of Control, Signals, and Systems, vol. 9, no. 2, pp. 95-106, 1996.

[34] R. Sepulchre, M. Jankovic, and P. Kokotovic, Constructive Nonlinear Control. Springer, 1997.

[35] A. Dietrich, Whole-Body Impedance Control of Wheeled Humanoid Robots, ser. Springer Tracts in Advanced Robotics. Springer International Publishing, 2016, vol. 116.

[36] A. Dietrich, C. Ott, and J. Park, "The Hierarchical Operational Space Formulation: Stability Analysis for the Regulation Case," IEEE Robotics and Automation Letters, vol. 3, no. 2, pp. 1120-1127, April 2018.

[37] A. Deo and I. Walker, "Overview of Damped Least-Squares Methods for Inverse Kinematics of Robot Manipulators," Journal of Intelligent Robotic Systems, vol. 14, no. 1, pp. 43-68, September 1995.

[38] A. Dietrich, T. Wimböck, A. Albu-Schäffer, and G. Hirzinger, "Integration of Reactive, Torque-Based Self-Collision Avoidance Into a Task Hierarchy," IEEE Transactions on Robotics, vol. 28, no. 6, pp. 12781293, December 2012.

[39] N. Dehio, D. Kubus, and J. J. Steil, "Continuously Shaping Projections and Operational Space Tasks," in Proc. of the 2018 IEEE/RSJ International Conference on Intelligent Robots and Systems, 2018.

[40] J. Lee, N. Mansard, and J. Park, "Intermediate Desired Value Approach for Task Transition of Robots in Kinematic Control," IEEE Transactions on Robotics, vol. 28, no. 6, pp. 1260-1277, December 2012.

[41] A. De Luca, A. Albu-Schäffer, S. Haddadin, and G. Hirzinger, "Collision Detection and Safe Reaction with the DLR-III Lightweight Manipulator Arm," in Proc. of the 2006 IEEE/RSJ International Conference on Intelligent Robots and Systems, October 2006, pp. 1623-1630.

[42] H. K. Khalil, Nonlinear Systems (Third Edition). Prentice Hall, 2002.

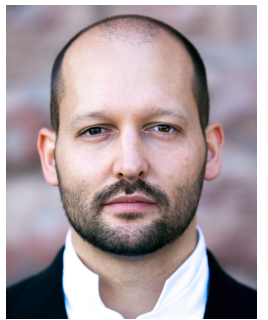

Alexander Dietrich received the doctoral degree from the Technical University of Munich (TUM), Germany, in 2015. He is currently head of the whole-body control group at the Institute of Robotics and Mechatronics of the German Aerospace Center (DLR). In 2016 he received the Georges Giralt Award for the best European PhD thesis in robotics. $\mathrm{He}$ is Editor of the IEEE International Conference on Robotics and Automation (ICRA), Associate Editor of the IEEE Robotics and Automation Magazine (RAM) and the IEEE Robotics and Automation Letters (RA-L), and he is co-chair of the IEEE RAS TC on Whole-Body Control. He currently holds a lecture at TUM on the control of modern lightweight robots. His research interests include robot control, humanoid robotics, mobile manipulation, and safe physical human-robot interaction.

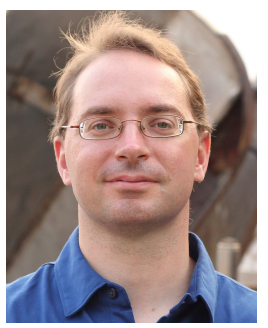

Christian Ott received the Dipl.-Ing. degree in mechatronics from Johannes Kepler University, Linz, Austria, in 2001 and the Dr.-Ing. degree in control engineering from Saarland University, Saarbruecken, Germany, in 2005. From 2001 to 2007, he was with the German Aerospace Center (DLR), Institute of Robotics and Mechatronics, Wessling, Germany. From May 2007 to June 2009, he was working as a project Assistant Professor in the Department of Mechano-Informatics, University of Tokyo, Tokyo, Japan. From 2011 to 2016 he has been working at DLR as a Team Leader of the Helmholtz Young Investigators Group for Dynamic Control of Legged Humanoid Robots. Since 2014, he is Head of the Department of Analysis and Control of Advanced Robotic Systems at DLR His current research interests include nonlinear robot control, flexible joint robots, impedance control, and control of humanoid robots.
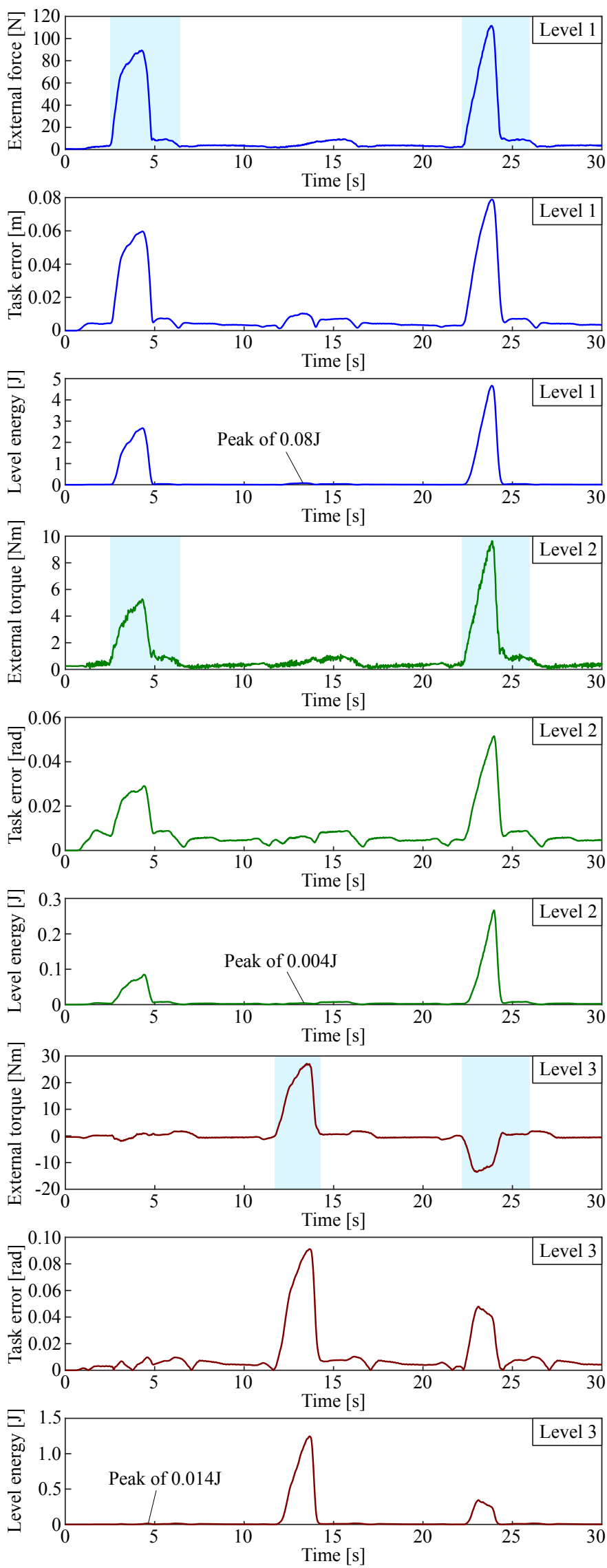

Fig. 20. Experiment \#2 (external disturbances): Control performance during physical interaction with the robot. 